\title{
Revenue Management Under a General Discrete Choice Model of Consumer Behavior
}

\author{
Kalyan Talluri * Garrett van Ryzin ${ }^{\dagger}$
}

October 21, 2001

\begin{abstract}
Customer choice behavior, such as "buy-up" and "buy-down", is an important phenomenon in a wide range of revenue management contexts. Yet most revenue management methodologies ignore this phenomenon - or at best approximate it in a heuristic way. In this paper, we provide an exact and quite general analysis of this problem. Specifically, we analyze a single-leg yield management problem in which the buyers' choice behavior is modeled explicitly. The choice model is perfectly general and simply specifies the probability of purchasing each fare product as function of the set of fare products offered. The control problem is to decide which subset of fare products to offer at each point in time. We show that the optimal policy is of a simple form. Namely, it consists of 1) identifying the ordered family of "nondominated" subsets $S_{1}, \ldots, S_{m}$, and 2 ) at each point in time opening one of these sets $S_{k}$, where the optimal index $k$ is increasing in the remaining capacity $x$. That is, the more capacity we have available, the further the optimal set is along this sequence. Moreover, we show that the optimal policy is nested if and only if the ordered sets are increasing, that is $S_{1} \subseteq S_{2} \subseteq \ldots \subseteq S_{n}$, and we give a complete characterization of when nesting by fare order is optimal. We then show that two important models, the independent demand model and the multinomial logit model (MNL), satisfy this later condition and hence nested-by-fare-order policies are optimal in these cases. We also develop an estimation procedure for this setting based on the expectation-maximization (EM) method that jointly estimates arrival rates and choice model parameters when no-purchase outcomes are unobservable. Numerical results are given to illustrate both the model and estimation procedure.
\end{abstract}

Key words. yield management, revenue management, discrete choice theory, airlines, dynamic programming, maximum likelihood estimation, EM method

\footnotetext{
*Kalyan Talluri, Department of Economics and Business, Universitat Pompeu Fabra, Ramon Trias Fargas 25-27, 08005 Barcelona, Spain, email: talluri@upf.es

${ }^{\dagger}$ Garrett van Ryzin, Graduate School of Business, Columbia University, New York, email:gjv1@columbia.edu
} 


\section{Introduction and Overview}

Yield (or revenue) management is a practice that dates back to the deregulation of the U.S. airline industry in the late 1970's. It was developed as an outgrowth of the need to manage capacity sold at discounted fares, which were targeted to leisure travelers, while simultaneously minimizing the dilution of revenue from business travelers willing and able to pay full fares. Using statistical forecasting techniques and mathematical optimization methods, airlines developed automated systems to dynamically control the availability of the many discounted fares that emerged in the post-deregulation era. The practice has since spread beyond airlines to the hospitality, rental-car, cruise-lines, railways, energy and broadcasting industries. Significant revenue benefits have been documented from such techniques - often an improvement of $2-8 \%$ in revenue over no revenue management or ad-hoc, manual controls [37].

Concurrent with the evolution of industry practice, a considerable amount of managementscience literature on yield management has been published over the last twenty years. The earliest work on capacity control was Littlewood's [28] analysis of a simple, two-fare-class model of capacity allocations on a single flight leg. The problem with more than two fare products (we define a fare product as a fare (rate, price) along with its associated set of restrictions to qualify for this fare) is considerably more complex, but Belobaba [5], [6], [7] developed two simple and effective heuristics for the single-leg problem based on the concept of expected marginal seat revenue (EMSR-a and EMSR-b) that are still in wide-spread use today.

On a theoretical level, single-leg models in which demand for each fare product is assumed to occur in non-overlapping periods have been developed and analyzed by Brumelle and McGill [13], Curry [16], Robinson [35] and Wollmer [45]. A key result of this work is that the optimal policy can be implemented using a set of so-called nested allocations. (See Brumelle and McGill [13] for a precise definition of nested allocations.) Lee and Hersh [26] introduced and analyzed a discrete-time, Markov model that allows for an arbitrary order of arrivals. For further work on single-leg allocation problems, see Brumelle et al. [14], Kleywegt and Papastavrou [24], Lautenbacher and Stidham [25], Liang [27], Stone and Diamond [38], Subramanian et al. [39] and Zhao [48]. For analysis of multiple-leg (network) allocation problems, see Cooper [15], Curry [16], Dror et al. [18], Glover et al. [22], Simpson [36], Talluri [40], Talluri and van Ryzin [41], [42] and Williamson [43], [44]. A recent surveys of yield management research is provided by McGill and van Ryzin [32]; Talluri and Barnhart [3] provide an overview of yield management and other airline operation research areas.

Despite the success of this body of work, most of the above-mentioned models make a common, simplifying - and potentially problematic - assumption; namely, that consumer demand for each of the fare products is completely independent of the controls being applied by the seller. That is, the problem is modeled as one of determining which exogenously arriving requests to accept or reject, and it is assumed that the likelihood of receiving a request for any given fare product does not depend on which other fares are available at 
the time of the request. However, casual observation - and a brief reflection on one's own buying behavior as a consumer - suggests that this is not the case in reality. The likelihood of selling a full fare ticket may very well depend on whether a discount fare is available at that time; the likelihood that a customer buys at all may depend on the lowest available fare, etc. Clearly, such behavior could have important revenue management consequences and should be considered when making control decisions.

We lay no claim to uncovering this deficiency. Indeed, many researches have tried to address "buy-up" (buying a higher fare when lower fares are closed) and "buy-down" (substituting a lower fare for a high fare when discounts are open) effects in the context of traditional models. Phillips [34] proposed a "state-contingent" approach to yield management that adjusts controls based on forecasts that depend on the controls in effect (the system "state") at any point in time. Belobaba [5] proposed a correction to the EMSR heuristics that introduces a probability of buying a higher fare when a low fare is closed. While conceptually appealing for a two-fare-class model, such pair-wise "buy-up" probabilities are problematic in a multiple-fare-class setting. The probability of buying a given high fare should depend on which other high fares are also available. Also, one cannot directly observe "buy-up", so how does one separate "original" sales from "buy-up" sales? How are the probabilities (forecasts) adjusted when there are price changes? etc. Despite the methodological difficulties, several airlines have experimented with consumer choice models for revenue management. The most significant is the work of Andersson [2] and Algers and Besser [1], who report a research and development effort at SAS to apply logit choice models to estimate buy-up and recapture factors at one of SAS's hubs.

Another stream of work on understanding choice behavior is the passenger origin and destination simulator (PODS) studies of Belobaba and Hopperstad. (See [9].) PODS is a detailed simulation model of passenger purchase behavior developed by Hopperstad and colleagues at Boeing. It includes factors for airline preference, time preference, path preference and price sensitivity. While it is a very detailed simulation model, the focus of the PODS studies is to test the performance of traditional forecasting and optimization methods under conditions of complex passenger choice behavior rather than to develop new estimation and optimization methods. Nevertheless, the PODS studies have provided many useful insights and clearly demonstrate the significant impact that choice behavior has on the performance of yield management systems.

The only theoretical models and methods that partially address choice behavior issues are dynamic pricing models, such as those studied by Bitran et al. [12], Feng and Gallego [19] and Gallego and van Ryzin [20], [21]. While these models allow demand to depend on the current price (the control in this case), they assume only one product is sold at one price at any point in time. Thus, customers face a binary choice; to buy or not to buy. In reality, firms offer many fares simultaneously and customers choose among them based on price together with their preferences for non-price factors, such as refundability and whether or not they can meet various restrictions (e.g. Saturday night stay, minimum-stay and maximum-stay). The above dynamic pricing models do not capture this complexity. 
In summary, while many attempts have been made to understand the impact of choice behavior on traditional yield management methods and to develop heuristics that partially capture buy-up and buy-down behavior, to date there is no methodology that directly and completely addresses the problem. In this paper, we develop a methodology that we believe substantially fills this void. We analyze a single-leg yield management problem in which we explicitly model consumer choice behavior using a general choice model, which specifies the probability of purchasing each fare product as a function of the set of available fare products. The model includes nearly every choice model of practical interest.

Given this general model of consumer choice behavior, we then formulate the single-leg, multiple-fare-class yield management problem as one of selecting a subset of fare products to offer at each point in time. We derive optimality conditions for the resulting dynamic program. While the policy might appear to be potentially complex under this model, we show that it has a simple form. First, we show that the optimal subsets can be reduced to an ordered family, $S_{1}, \ldots, S_{m}$, of nondominated subsets (the definition of a nondominated subset is defined precisely below). Typically, this family of subsets is much smaller than the number of total possible subsets. The optimal policy then consists of opening one of the sets $S_{k}$ in the sequence, where the optimal index $k$ is increasing in the remaining capacity $x$. That is, the more capacity we have available at any point in time, the further the optimal set is along the sequence. Moreover, we show that the optimal policy is a nested allocation policy (defined precisely below) if and only if the family of nondominated subsets is increasing that is $S_{1} \subseteq S_{2} \subseteq \ldots \subseteq S_{m}$. This provides a very complete and general characterization of the cases in which nested allocation policies are optimal. We also provide conditions that gaurantee the nesting is by fare class order. We use these conditions to show that for the traditional, independent-demand model, the optimal policy is nested by fare class order. The same conditions show that for the classical multinomial logic (MNL) choice model, the optimal policy is nested by fare class order as well.

We also develop a practical estimation procedure for our model. One major difficulty in estimating choice models in the yield management setting is that one typically cannot observe no-purchase decisions. In many industries, sale are conducted remotely and anonymously and the only available data are purchase transactions. Thus, it is often impossible to distinguish between periods with no arrival and periods in which there was an arrival and the arriving customer decided not to purchase. (An exception is when sales are direct, e.g. from the firm's own web site, in which case considerable information on no-purchases can potentially be gathered). We overcome this incomplete data problem by applying the expectation-maximization (EM) method of Dempster et al. [17] to the traditional maximumlikelihood discrete-choice parameter estimation. The method allows us to simultaneously estimate both the parameters of the choice model and the arrival rates using only transaction data on sales. Together, our estimation procedure and optimization model provide a theoretically sound and quite complete approach to the single-leg problem with choice behavior.

The remainder of the paper is organized as follows: In Section 1 we define the choicebased model of the problem. In Section 2 we formulate a dynamic program and analyze the 
resulting optimal policy and Section 3 looks at the optimality of nested policies. Section 4 describes our EM-based estimation procedure. Finally, some brief numerical examples are given in Section 5 and our conclusions are given in Section 6.

\section{Model}

Time is discrete and indexed by $t$, and the indices run backwards in time (e.g. smaller values of $t$ represent later points in time). Time $t=0$ represents the deadline for the sale of units. In each period there is at most one arrival. The probability of arrival is denoted by $\lambda$, which we assume is the same for all time periods $t$. While extending the results to time-varying arrival probabilities is straightforward, it is cumbersome and we omit the details to simplify the exposition. There are $n$ fare products and $N=\{1, \ldots, n\}$ denotes the entire set of fare products. Each fare product $j \in N$ has an associated revenue (fare) $r_{j}$, and without loss of generality we index fare products so that $r_{1} \geq r_{2} \geq \ldots r_{n} \geq 0$.

In each period $t$, the firm must choose a subset $S \subseteq N$ of fare products to offer. When the fares $S$ are offered, the probability that a customer chooses class $j \in S$ is denoted $P_{j}(S)$ and we assume $P_{j}(S)=0$ if $j \notin S$. We let $j=0$ denote the no-purchase choice; that is, the event that the customer does not purchase any of the fares offered in $S . P_{0}(S)$ denotes the no-purchase probability. It is possible to allow the choice probabilities to be a function of time $t$ as well, but to keep the notation simple we assume that the probabilities do not depend on time. The probability that a sale of class $j$ is made in period $t$ is therefore $\lambda P_{j}(S)$, and the probability that no sale is made is $\lambda P_{0}(S)+(1-\lambda)$. Note this last expression reflects the fact that having no sales in a period could be due either to no arrival at all or an arrival that does not purchase; as mentioned, this leads to an incomplete data problem when estimating the model.

The only conditions we impose on the choice probabilities $P_{j}(S)$ it that they define a proper probability function. That is, for every set $S \subseteq N$, the probabilities satisfy $P_{j}(S) \geq 0$ for all $j \in S$ and $\sum_{j \in S} P_{j}(S)+P_{0}(S)=1$. This includes most all choice models of interest. For example, some psychologists shown that customers can be overwhelmed by more choices, and they may become more reluctant to purchase as more options are offered (See Iyengar [23].). Such cases would be covered by a suitable choice of $P_{j}(S)$ that is decreasing in $S$. It also includes all discrete choice models used in practice, such as those described in BenAkiva and Lerman [10]. The only real limitation is that we assume the choices are only a function of the set $S$ of open fares at the time of purchase. In particular, we do not model potential strategic behavior (e.g. when a buyer's choice depends on the seller's policy or the strategies of other buyers) or history-dependent choice behavior (e.g. when a buyer's choice depends on his/her past choices or past events in the system).

We will use the following running example to illustrate the model and analysis:

Example 1: An airline offers three fare products, $Y, M$ and $Q$. These classes differ in terms of revenues and conditions as shown in Table 1. The airline has 5 segments of customers, 2 


\begin{tabular}{c|ccc} 
Fare Product (Class) & SA Stay & 21-Day Adv. & Revenue \\
\hline$Y$ & No & No & $\$ 800$ \\
$M$ & No & Yes & $\$ 500$ \\
$Q$ & Yes & Yes & $\$ 450$
\end{tabular}

Table 1: Fare product revenues and restrictions for Example 1

\begin{tabular}{c|c|cccc} 
& & \multicolumn{2}{|c}{ Qualifies for restrictions? } & \multicolumn{2}{c}{ Willing to buy? } \\
Segment & Prob. & SA Stay & 21-Day Adv. & $Y$ Class & $M$ Class \\
\hline Bus. 1 & 0.1 & No & No & Yes & Yes \\
Bus. 2 & 0.2 & No & Yes & Yes & Yes \\
Leis. 1 & 0.2 & Yes & No & No & Yes \\
Leis. 2 & 0.2 & Yes & Yes & No & Yes \\
Leis. 3 & 0.3 & Yes & Yes & No & No
\end{tabular}

Table 2: Segments and their characteristics for Example 1

business segments and 3 leisure segments. The segments differ in terms of the restrictions that they qualify for and the fares they are willing to pay. The data describing each segment are given in Table 2. The second column of Table 2 gives the probability that an arriving customer is from each given segment.

Given these data for Example 1, Table 3 describes the choice probabilities that would result. To see how the probabilities in Table 3 are derived, consider the set $S=\{Y, Q\}$. If $S=\{Y, Q\}$ is offered, segments Business 1 and Business 2 buy the $Y$ fare, because they cannot qualify for both the SA stay and 21-day advance restrictions on $Q$, so $P_{Y}=$ $0.1+0.2=0.3$. Similarly, Leisure 1 cannot qualify for the 21 day advance restriction of $Q$ and is not willing to purchase $Y$, so these customers do not purchase at all. Segments Leisure 2 and 3, however, qualify for both restrictions on $Q$ and purchase $Q$. Hence, $P_{Q}=0.2+0.3=0.5$. Class $M$ is not offered, so $P_{M}=0$. The other rows of Table 3 are filled out similarly.

Again, this particular method of generating choice probabilities is only for illustration. Other choice models could be used and in general any proper set of probabilities could be used to populate Table 3 .

\section{Optimization}

We next formulate a single-leg problem based on this general choice model. Let $C$ denote the aircraft capacity, $T$ denote the number of time periods, $t$ denote the number of remaining 


\begin{tabular}{l|rrr|rr|c}
$S$ & $P_{Y}(S)$ & $P_{M}(S)$ & $P_{Q}(S)$ & $\mathrm{Q}(\mathrm{S})$ & $\mathrm{R}(\mathrm{S})$ & Dominated? \\
\hline$\{Y\}$ & 0.3 & 0 & 0 & 0.3 & 240 & No \\
$\{M\}$ & 0 & 0.4 & 0 & 0.4 & 200 & Yes \\
$\{Q\}$ & 0 & 0 & 0.5 & 0.5 & 225 & Yes \\
$\{Y, M\}$ & 0.1 & 0.4 & 0 & 0.5 & 280 & Yes \\
$\{Y, Q\}$ & 0.3 & 0 & 0.5 & 0.8 & 465 & No \\
$\{M, Q\}$ & 0 & 0.4 & 0.5 & 0.9 & 425 & Yes \\
$\{Y, M, Q\}$ & 0.1 & 0.4 & 0.5 & 1 & 505 & No
\end{tabular}

Table 3: Choice probabilities $P_{j}(S)$, probability of purchase, $Q(S)$ and expected revenue, $R(S)$ for Example 1

periods (recall time is indexed backwards) and $x$ denotes the number of remaining inventory units. Define the value function $V_{t}(x)$ as the maximum expected revenue obtainable from periods $t, t-1, \ldots, 1$ given that there are $x$ inventory units remaining at time $t$. Then the Bellman equation for $V_{t}(x)$ is

$$
\begin{aligned}
V_{t}(x) & =\max _{S \subseteq N}\left\{\sum_{j \in S} \lambda P_{j}(S)\left(r_{j}+V_{t-1}(x-1)\right)+\left(\lambda P_{0}(S)+1-\lambda\right) V_{t-1}(x)\right\} \\
& =\max _{S \subseteq N}\left\{\sum_{j \in S} \lambda P_{j}(S)\left(r_{j}-\Delta V_{t-1}(x)\right)\right\}+V_{t-1}(x),
\end{aligned}
$$

where $\Delta V_{t-1}(x)=V_{t-1}(x)-V_{t-1}(x-1)$ denotes the marginal cost of capacity, and we have used the fact that for all $S$,

$$
\sum_{j \in S} P_{j}(S)+P_{0}(S)=1
$$

The boundary conditions are

$$
V_{t}(0)=0, \quad t=1, \ldots, T \text { and } V_{0}(x)=0, \quad x=1, \ldots, C .
$$

We can write (1) in more compact form as

$$
V_{t}(x)=\max _{S \subseteq N}\left\{\lambda\left(R(S)-Q(S) \Delta V_{t-1}(x)\right)\right\}+V_{t-1}(x),
$$

where

$$
Q(S)=\sum_{j \in S} P_{j}(S)=1-P_{0}(S)
$$

denotes the total probability of purchase and

$$
R(S)=\sum_{j \in S} P_{j}(S) r_{j}
$$


denotes the total expected revenue from offering set $S$. Table 3 gives the values $Q(S)$ and $R(S)$ for our Example 1.

A sequence of sets achieving the maximum in (3) forms an optimal Markovian policy ( $c f$. Bellman [4] and Bertsekas [11]). For theoretical purposes, we will also consider allowing the seller to randomize over the sets $S$ that are offered at the beginning of each time period. Since the number of subsets is finite, there is always one set $S$ that maximizes $\lambda\left(R(S)-Q(S) \Delta V_{t-1}(x)\right)$ (there may be ties or course), so randomizing among the sets to offer provides no additional benefit to the seller (at most they can randomize between two or more optimal sets and achieve the same revenue as using one of the optimal sets alone). However, allowing this flexibility in policies will be useful theoretically.

Potentially, each optimization on the right hand side of (3) could require an evaluation of all $2^{n}$ subsets. However, we show next that the search can be reduced to an evaluation of only nondominated sets. These sets are defined as follows:

Definition $1 A$ set $T$ is said to be dominated if there exist probabilities $\alpha(S), \forall S \subseteq N$ with $\sum_{S \subseteq N} \alpha(S)=1$ such that either

$$
Q(T) \geq \sum_{S \subseteq N} \alpha(S) Q(S) \quad \text { and } \quad R(T)<\sum_{S \subseteq N} \alpha(S) R(S)
$$

or

$$
Q(T)>\sum_{S \subseteq N} \alpha(S) Q(S) \quad \text { and } \quad R(T) \leq \sum_{S \subseteq N} \alpha(S) R(S) .
$$

A set is said to be nondominated if it is not dominated.

In words, a set $T$ is dominated if we can use a randomization of other sets $S$ to produce an expected revenue that is strictly greater than $R(T)$ with no increase in the probability of purchase $Q(T)$ (or the same revenue $R(T)$ with a probability strictly lower than $Q(T)$ ). For Example 1, Table 3 shows which sets are nondominated, namely the sets $\{Y\},\{Y, Q\}$ and $\{Y, Q, M\}$. That these sets are nondominated follows from inspection of Figure 1, which shows a scatter plot of the value $Q(S)$ and $R(S)$ for all subsets $S$. Note from this figure and Definition 1, that a nondominated set is a point that is on the "efficient frontier" of the set of points $\{Q(S), R(S)\}, S \subseteq N$, a concept that should be natural to those readers familiar with mean-variance portfolio theory or data envelopment analysis (DEA). Here, "efficiency" is with respect to the trade-off between expected revenue, $R(S)$, and probability of sale, $Q(S)$.

An alternative characterization of a nondominated set, which is useful analytically, is given by the following proposition:

Proposition $1 A$ set $T$ is nondominated if and only if, for some value $v>0, T$ is an optimal solution to

$$
\max _{S \subset N}\{R(S)-v Q(S)\}
$$




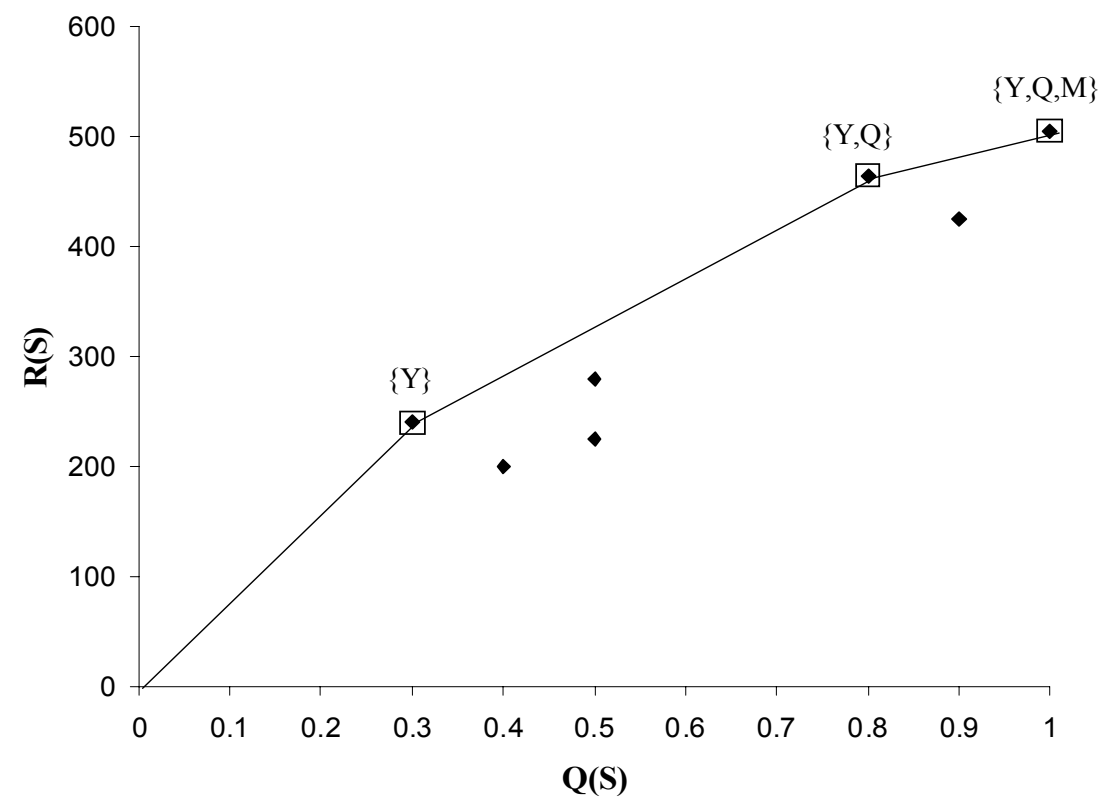

Figure 1: Scatter plot of $Q(S)$ and $R(S)$ for Example 1 (nondominated points are enclosed in squares and labeled)

$\underline{\text { Proof }}$

We first show the "if" part: Suppose for some $v>0, T$ satisfies $R(T)-v Q(T) \geq R(S)-$ $v Q(S)$ for all $S \subseteq N$. This is equivalent to

$$
(R(T)-R(S))-v(Q(T)-Q(S)) \geq 0, \quad \forall S \subseteq N
$$

Multiplying each inequality above by $\alpha(S)$ where $\{\alpha(S): S \subseteq N\}$ are any set of probabilities with $\sum_{S \subseteq N} \alpha(S)=1$ and adding the inequalities, we then have that

$$
\left(R(T)-\sum_{S \subseteq N} \alpha(S) R(S)\right)-v\left(Q(T)-\sum_{S \subseteq N} \alpha(S) Q(S)\right) \geq 0 .
$$

Now if $v>0$ and $T$ is dominated, there exists probabilities $\alpha(S)$ such that $R(T)<$ $\sum_{S \subseteq N} \alpha(S) R(S)$ and $Q(T) \geq \sum_{S \subseteq N} \alpha(S) Q(S)$ (or for which $R(T) \leq \sum_{S \subseteq N} \alpha(S) R(S)$ and $Q(T)>\sum_{S \subseteq N} \alpha(S) Q(S)$ ), which contradicts the above inequality. Therefore $T$ must be nondominated. This proves the "if" part.

To show the "only if" part, we will show that if $T$ is nondominated, there exists a value $v^{*}>0$ such that $T$ maximizes $R(S)-v^{*} Q(S)$ over $S \subseteq N$. Since $T$ is nondominated, we 
have

$$
\begin{aligned}
R(T) \geq \quad & \max \sum_{S \subseteq N} \alpha(S) R(S) \\
& \sum_{S \subseteq N} \alpha(S) Q(S) \leq Q(T) \\
& \sum_{S \subseteq N} \alpha(S)=1 \\
& \alpha(S) \geq 0, \quad S \subseteq N .
\end{aligned}
$$

If we relax the constraint $\sum_{S \subseteq N} \alpha(S) Q(S) \leq Q(T)$ by adding a Lagrange multiplier $v$ we have by weak duality

$$
R(T) \geq \min _{v \geq 0} z_{d}(v)
$$

where the dual function $z_{d}(v)$ is defined by

$$
\begin{aligned}
z_{d}(v)= & \max \sum_{S \subseteq N} \alpha(S)[R(S)-v Q(S)]+v Q(T) \\
& \sum_{S \subseteq N} \alpha(S)=1 \\
& \alpha(S) \geq 0, S \subseteq N
\end{aligned}
$$

Since the primal (4) is feasible and bounded and the dual (6) is always feasible, by strong duality for linear programs there exists an optimal dual solution $v^{*} \geq 0$ such that $R(T)=$ $z_{d}\left(v^{*}\right)$. Let $\alpha^{*}(\cdot)$ denote the optimal solution to (6) for this value $v^{*}$. Thus, we have

$$
R(T)-v^{*} Q(T)=\sum_{S \subseteq N} \alpha^{*}(S) R(S)-v^{*} \sum_{S \subseteq N} \alpha^{*}(S) Q(S)
$$

We will show that $T$ maximize $R(S)-v^{*} Q(S)$ over $S \subseteq N$. Indeed, suppose it does not; then there exist a $S^{*}$ with $R\left(S^{*}\right)-v^{*} Q\left(S^{*}\right)>R(T)-v^{*} Q(T)$ and hence

$$
\begin{aligned}
R\left(S^{*}\right)-v^{*}\left(Q\left(S^{*}\right)-Q(T)\right) & >R(T) \\
& \geq \sum_{S \subseteq N} \alpha^{*}(S)\left[R(S)-v^{*} Q(S)\right]+v^{*} Q(T) .
\end{aligned}
$$

But this contradicts the fact that $\alpha^{*}(S)$ maximizes (6). Therefore $T$ maximizes $R(S)-$ $v^{*} Q(S)$.

We next show there always exists such a $v^{*}>0$. To do so, suppose $v^{*}=0$ is the unique dual solution. Note that in this case $z_{d}(0)=\max _{S \subseteq N}\{R(S)\}$, which implies that $R(T)=\max _{S \subseteq N}\{R(S)\}$. Since $v^{*}=0$ is the unique solution to the dual, we must have that for all $v>0, R(T)=z_{d}(0)<z_{d}(v)$ (else $v^{*}=0$ is not the unique minimizer of the dual). But by (6) this in turn implies that for all $v>0$,

$$
R(T)-v Q(T)<\sum_{S \subseteq N} \alpha^{*}(S)[R(S)-v Q(S)]
$$


where $\alpha^{*}(\cdot)$ is the optimal solution to $(6)$, or equivalently

$$
\left(R(T)-\sum_{S \subseteq N} \alpha^{*}(S) R(S)\right)-v\left(Q(T)-\sum_{S \subseteq N} \alpha^{*}(S) Q(S)\right)<0 .
$$

Now since $R(T)=\max _{S \subseteq N}\{R(S)\}$, the first term is nonnegative and since $v>0$, this means we must have $Q(T)>\sum_{S \subseteq N} \alpha^{*}(S) Q(S)$. But this contradicts the fact that $T$ is nondominated. Therefore $v^{*}=0$ can never be the unique dual solution, so we can assume there always exists a $v^{*}>0$. This completes the "only if" proof.

The significance of dominated sets is that they can be eliminated from consideration from our optimization problem, as shown by the following proposition:

Proposition 2 If $\Delta V_{t-1}(x)>0$, then a dominated set is never an optimal solution to (1). If $\Delta V_{t-1}(x)=0$, then there always exists a nondominated set $T$ that is an optimal solution to (1).

$\underline{\text { Proof }}$

The proof for the case $\Delta V_{t-1}(x)>0$ follows directly from Proposition 1. For the case $\Delta V_{t-1}(x)=0$, note that an optimal solution $T$ to (1) must maximize the expected revenue, i.e. satisfy $R(T)=\max _{S \subseteq N}\{R(S)\}$. But it is not hard to see from Proposition 1 that there must be at least one such $T$ that is nondominated.

It will sometimes be useful to have a slightly stronger notion of dominance:

Definition $2 A$ set $T$ is said to be strongly nondominated if $T$ is the unique solution to $\max _{S \subset N}\{R(S)-v Q(S)\}$ for some value $v \geq 0$.

A set that is nondominated but not strongly nondominated could also be eliminated, because it is never a unique optimal solution. However, this elimination has to be done with more care since eliminating one such set might make another set strongly nondominated relative to the remaining sets. In contrast, all dominated sets can be eliminated without checking the effect on other sets.

We next show that the nondominated sets are used in a quite simple order. Indeed, let $m$ denote the number of nondominated sets. These sets can be indexed $S_{1}, \ldots, S_{m}$ such that both the revenues and probabilities of purchase are monotone increasing in the index:

Proposition 3 If the collection of $m$ nondominated sets is indexed such that $Q\left(S_{1}\right) \leq$ $Q\left(S_{2}\right) \leq \ldots \leq Q\left(S_{m}\right)$, then $R\left(S_{1}\right) \leq R\left(S_{2}\right) \leq \ldots \leq R\left(S_{m}\right)$ as well.

$\underline{\text { Proof }}$

The proof is by contradiction. Suppose we order the sets so that $Q\left(S_{k}\right)$ is increasing but 
there exist an $l<k$ in this ordering such that $R\left(S_{l}\right)>R\left(S_{k}\right)$. (There has to be at least one such $l$ and $k$ else the sequence is ordered as claimed.) For this $l$ and $k$, we have $R\left(S_{l}\right)>R\left(S_{k}\right)$ and $Q\left(S_{l}\right) \leq Q\left(S_{k}\right)$, which means $S_{k}$ is trivially dominated by $S_{l}$. But this is a contradiction, so we must have that $R\left(S_{k}\right)$ is increasing in $k$ as well.

For Example 1, we see from Table 3 that there are $m=3$ nondominated sets $\{Y\}$, $\{Y, Q\}$ and $\{Y, Q, M\}$. These can be ordered $S_{1}=\{Y\}, S_{2}=\{Y, Q\}$ and $S_{3}=\{Y, Q, M\}$ with associated probabilities of purchase $Q_{1}=0.3, Q_{2}=0.8$ and $Q_{3}=1$ and revenue $R_{1}=\$ 240, R_{2}=\$ 465$ and $R_{3}=\$ 505$ as claimed.

Henceforth, we assume the nondominated sets are denoted $S_{1}, \ldots, S_{m}$ and are indexed in increasing revenue and probability order. Also, to keep notion simpler we let $R_{k}=R\left(S_{k}\right)$ and $Q_{k}=Q\left(S_{k}\right)$, and note $R_{k}$ and $Q_{k}$ are both increasing in $k$. So the Bellman equation can be further simplified to

$$
V_{t}(x)=\max _{k=1, \ldots, m}\left\{\lambda\left(R_{k}-Q_{k} \Delta V_{t-1}(x)\right)\right\}+V_{t-1}(x),
$$

When expressed in terms of the sequence $S_{1}, \ldots, S_{m}$ of nondominated sets, we show next that the optimal policy has a quite simple form. We first need some preliminary lemmas.

Lemma 1 Let $l>k$ be two indexes of nondominated sets and suppose that $R_{l}-Q_{l} v_{0}>$ $R_{k}-Q_{k} v_{0}$ for some nonnegative $v_{0}$. Then, $R_{l}-Q_{l} v>R_{k}-Q_{k} v$ for all $v \leq v_{0}$.

$\underline{\text { Proof }}$

Restating the condition, we want to show that if

$$
R_{l}-R_{k}>\left(Q_{l}-Q_{k}\right) v
$$

holds for $v=v_{0}$ it holds for all $v \leq v_{0}$, but this follows trivially from the fact that if $l>k$, $R_{l}-R_{k} \geq 0$ and $Q_{l}-Q_{k} \geq 0$ so the RHS above is increasing in $v$.

The next lemma shows that the index of the optimal nondominated set in (7) is decreasing in the value $\Delta V_{t-1}(x)$

Lemma 2 Let $k^{*}$ denote the index of the nondominated set $S_{k^{*}}$ that maximizes (7) (or greatest such index if more than one nondominated set maximizes (7)). Then $k^{*}$ is decreasing in $\Delta V_{t-1}(x)$.

$\underline{\text { Proof }}$

For ease of notation, let $v=\Delta V_{t-1}(x)$ and note that the optimal index $k$ solves

$$
\max _{k=1, . ., m}\left\{R_{k}-Q_{k} v\right\}
$$


For $v=0$, it is clear that $k=m$ is optimal since $R_{m}$ is the largest revenue (or if there is a tie, $k=m$ is the largest index of the sets with maximum revenue). Now consider increasing $v$ from zero. As $v$ is increased, as some value $v_{1}$ a new index $k_{1}$ will become the smallest optimal index, and clearly $k_{1}<m$. Increasing $v$ further we reach another value $v_{2}$ where a new index $k_{2}$ becomes the largest optimal index. We will show that $k_{2}<k_{1}$. Indeed, suppose that $k_{2}>k_{1}$. Then by Lemma 1 , we have that

$$
R_{k_{2}}-Q_{k_{2}} v>R_{k_{1}}-Q_{k_{1}} v, \quad \forall v \leq v_{2}
$$

but this contradicts the fact that $k_{1}$ is optimal for $v_{1} \leq v<v_{2}$. Therefore, we must have that $k_{2}<k_{1}$. Repeating this argument as $v$ is increased shows that the largest optimal index $k^{*}(v)$ is always decreasing in $v$.

Our next lemma shows that the marginal value is decreasing in the remaining capacity.

Lemma $3 \Delta V_{t}(x) \leq \Delta V_{t}(x-1), \quad t=1, \ldots, T, \quad x=1, \ldots, C$

$\underline{\text { Proof }}$

The proof is by induction on $t$. First, the statement is trivially true for $t=0$ by the boundary conditions (2). Assume it is true for period $t-1$. Let $S_{t}^{*}(x)$ denote the optimal solution to (1) and note

$$
\begin{aligned}
\Delta V_{t}(x)-\Delta V_{t}(x-1)= & \left(\Delta V_{t-1}(x)-\Delta V_{t-1}(x-1)\right) \\
& +\sum_{j \in S_{t}^{*}(x)} \lambda P_{j}\left(S_{t}^{*}(x)\right)\left(r_{j}-\Delta V_{t-1}(x)\right) \\
& -\sum_{j \in S_{t}^{*}(x-1)} \lambda P_{j}\left(S_{t}^{*}(x-1)\right)\left(r_{j}-\Delta V_{t-1}(x-1)\right) \\
& -\sum_{j \in S_{t}^{*}(x-1)} \lambda P_{j}\left(S_{t}^{*}(x-1)\right)\left(r_{j}-\Delta V_{t-1}(x-1)\right) \\
& +\sum_{j \in S_{t}^{*}(x-2)} \lambda P_{j}\left(S_{t}^{*}(x-2)\right)\left(r_{j}-\Delta V_{t-1}(x-2)\right)
\end{aligned}
$$

From the optimality of the set defined by $S_{t}^{*}(\cdot)$, the following inequalities hold:

$$
\sum_{j \in S_{t}^{*}(x-1)} \lambda P_{j}\left(S_{t}^{*}(x-1)\right)\left(r_{j}-\Delta V_{t-1}(x-1)\right) \geq \sum_{j \in S_{t}^{*}(x)} \lambda P_{j}\left(S_{t}^{*}(x)\right)\left(r_{j}-\Delta V_{t-1}(x-1)\right)
$$

and

$$
\sum_{j \in S_{t}^{*}(x-1)} \lambda P_{j}\left(S_{t}^{*}(x-1)\right)\left(r_{j}-\Delta V_{t-1}(x-1)\right) \geq \sum_{j \in S_{t}^{*}(x-2)} \lambda P_{j}\left(S_{t}^{*}(x-2)\right)\left(r_{j}-\Delta V_{t-1}(x-1)\right)
$$

Substituting into (8) we obtain

$$
\Delta V_{t}(x)-\Delta V_{t}(x-1) \leq\left(\Delta V_{t-1}(x)-\Delta V_{t-1}(x-1)\right)
$$




$$
\begin{aligned}
& +\sum_{j \in S_{t}^{*}(x)} \lambda P_{j}\left(S_{t}^{*}(x)\right)\left(r_{j}-\Delta V_{t-1}(x)\right) \\
& -\sum_{j \in S_{t}^{*}(x)} \lambda P_{j}\left(S_{t}^{*}(x)\right)\left(r_{j}-\Delta V_{t-1}(x-1)\right) \\
& -\sum_{j \in S_{t}^{*}(x-2)} \lambda P_{j}\left(S_{t}^{*}(x-2)\right)\left(r_{j}-\Delta V_{t-1}(x-1)\right) \\
& +\sum_{j \in S_{t}^{*}(x-2)} \lambda P_{j}\left(S_{t}^{*}(x-2)\right)\left(r_{j}-\Delta V_{t-1}(x-2)\right)
\end{aligned}
$$

Rearranging and canceling terms yields

$$
\begin{aligned}
\Delta V_{t}(x)-\Delta V_{t}(x-1) \leq & \left(1-\sum_{j \in S_{t}^{*}(x)} \lambda P_{j}\left(S_{t}^{*}(x)\right)\right)\left(\Delta V_{t-1}(x)-\Delta V_{t-1}(x-1)\right) \\
& +\sum_{j \in S_{t}^{*}(x-2)} \lambda P_{j}\left(S_{t}^{*}(x-2)\right)\left(\Delta V_{t-1}(x-1)-\Delta V_{t-1}(x-2)\right)
\end{aligned}
$$

By induction, $\Delta V_{t-1}(x)-\Delta V_{t-1}(x-1) \leq 0$ and $\Delta V_{t-1}(x-1)-\Delta V_{t-1}(x-2) \leq 0$. Therefore, $\Delta V_{t}(x)-\Delta V_{t}(x-1) \leq 0$.

Thus, marginal values are decreasing, which is intuitive. (Nonmonotonicty of the marginal values could occur if there is demand for multiple inventory units (group requests); See for example Kleywegt and Papastavrou [24], Lee and Hersh [26] and Young and Van Slyke $[47])$.

The marginal values are increasing in the remaining time as well:

Lemma $4 \Delta V_{t}(x) \geq \Delta V_{t-1}(x), \quad t=1, \ldots, T, \quad x=1, \ldots, C$

$\underline{\text { Proof }}$

Note that by using (7), we have

$$
\begin{aligned}
\Delta V_{t}(x) & =V_{t}(x)-V_{t}(x-1) \\
& =\max _{k}\left\{\lambda\left(R_{k}-Q_{k} \Delta V_{t-1}(x)\right)\right\}-\max _{k}\left\{\lambda\left(R_{k}-Q_{k} \Delta V_{t-1}(x-1)\right)\right\}+\Delta V_{t-1}(x) .
\end{aligned}
$$

From Lemma $3, \Delta V_{t-1}(x) \leq \Delta V_{t-1}(x)$, and therefore for any value $k$,

$$
\lambda\left(R_{k}-Q_{k} \Delta V_{t-1}(x)\right)-\lambda\left(R_{k}-Q_{k} \Delta V_{t-1}(x)\right) \geq 0 .
$$

Hence

$$
\max _{k}\left\{\lambda\left(R_{k}-Q_{k} \Delta V_{t-1}(x)\right)\right\}-\max _{k}\left\{\lambda\left(R_{k}-Q_{k} \Delta V_{t-1}(x)\right)\right\} \geq 0
$$

as well, and it follows that $\Delta V_{t}(x) \geq \Delta V_{t-1}(x)$.

By combining Lemmas 2, 3 and 4, we obtain our main theorem: 
Theorem 1 An optimal policy for (1) is to select a set $k^{*}$ from among the $m$ nondominated, ordered sets $\left\{S_{k}: k=1, \ldots, m\right\}$ that maximizes (7). Moreover, for a fixed $t$, the largest optimal index $k^{*}$ is increasing in the remaining capacity $x$, and for any fixed $x, k^{*}$ is decreasing in the remaining time $t$.

This characterization is significant for several reasons. First, it shows that the optimal sets can be reduced to only those that are nondominated, which in many cases significantly reduces the number of sets we need to consider. Moreover, it shows that these limited number of sets can be sequenced in a natural way and that the more capacity we have (or the less time remaining), the higher the set we should use in this sequence. For example, applying Theorem 1 to Example 1, we see that the nondominated sets $S_{1}=\{Y\}, S_{2}=$ $\{Y, Q\}$ and $S_{3}=\{Y, Q, M\}$ would be used as follows: with very large amounts of capacity remaining, $S_{3}$ is optimal - i.e. all three fare classes are opened. As capacity is consumed, at some point we switch to only offering $S_{2}$ - i.e. Class $M$ is closed and only $Y$ and $Q$ are offered. As capacity is reduced further, at some point we close Class $M$ and only offer Class $Y$ (i.e. set $S_{1}$ is used).

\section{Optimality of nested allocation policies}

The optimization results above have significant implications for the optimality of nested allocation policies. The notion of dominance from Definition 1 and Theorem 1 can be used to provide a quite complete characterization of cases in which nested allocation polices are optimal. They also can be used to provide conditions under which the optimal nesting is by fare order.

\subsection{General nested policies}

To begin, we first need to precisely define a nested allocation policy.

Definition 3 A control policy for (1) is called a nested policy if there is an increasing

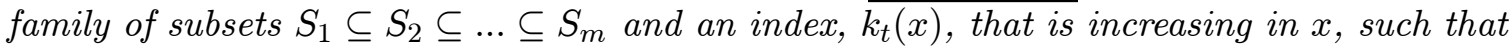
set $S_{k_{t}(x)}$ is chosen at time $t$ when the remaining capacity is $x$.

Though this is a somewhat abstract definition of a nested policy, it is in fact the natural generalization of nested allocations from the traditional single-leg models. In particular, it implies an ordering of the classes based on when they first appear in the increasing sequence of sets $S_{k}$. That is, class $i$ is considered "higher" than class $j$ in the nesting order if class $i$ appears earlier in the sequence. Returning to Example 1, we see that the the nondominated sets are indeed nested according to this definition because $S_{1}=\{Y\}$, $S_{2}=\{Y, Q\}$ and $S_{3}=\{Y, Q, M\}$ are increasing. Class $Y$ would be considered the highest in the nested order, followed by Class $Q$ and then Class $M$. 
We will say a policy is nested by fare order if the nesting order is the same as the fare order. Note that in Example 1 the nested order is not the fare order, since $M$ is the lowest ranked fare product in nested order but $Q$ has the lowest revenue.

If the optimal policy is nested one can use a nested allocation policy to implement it. A nested allocation policy is defined as follows: for each set $S_{k}$ we can define a set of nested protection levels, $p_{k}, k=1, . ., m$, with $p_{1} \leq p_{2} \leq \ldots \leq p_{m}$ such that classes lower than $S_{k}$ are closed if the remaining capacity is less than $p_{k}$. The protection levels are defined by

$$
p_{k}=\max \left\{x: R_{k}-Q_{k} \Delta V_{t-1}(x)>R_{k+1}-Q_{k+1} \Delta V_{t-1}(x)\right\}, \quad k=1,2, . . m-1
$$

where for notational convenience we define $p_{0}=0$ and $p_{m}=C$. The set $S_{k}$ should be used if and only if $p_{k} \geq x>p_{k-1}$ and the classes $S_{m}-S_{k}$ should be closed if $x \leq p_{k}$. That is, there is a critical threshold of capacity below which we close off fares in $S_{m}-S_{k}$.

We can also define nested booking limits for Class $i, b_{i}$ as follows: Let $k(i)$ denote the index of the first set in which Class $i$ appears in the sequence of nondominated sets. Then the booking limit

$$
b_{i}=C-p_{k(i)-1}, \quad i=1, \ldots, n
$$

where $p_{k(i)-1}$ is the protection limit for the set $S_{k(i)-1}$.

We again return to Example 1 to illustrate this concept. Table 4 shows the objective function value $R_{k}-Q_{k} \Delta V_{t-1}(x)$ for each of the three nondominated set sets $k=1,2,3$ (recall $S_{1}=\{Y\}, S_{2}=\{Y, Q\}$ and $S_{3}=\{Y, Q, M\}$ ) for a particular marginal value function $\Delta V_{t-1}(x)$. Capacities are in the range $x=1,2, \ldots, 20$. The last column of Table 4 gives the index, $k_{t}^{*}(x)$, of the nondominated set that is optimal for each capacity $x$. Note that for capacities 1,2 and 3, the set $S_{1}=\{Y\}$ is the optimal set, so Class $Y$ is the only open fare. Once we reach 4 units of remaining capacity, set $S_{2}=\{Y, Q\}$ becomes optimal and we open Classes $Q$ in addition to Class $Y$. When the remaining capacity reaches 13 , set $S_{3}=\{Y, Q, M\}$ becomes optimal, and we open $M$ in addition to $Y$ and $Q$. As a result, the protection level for set $S_{1}$, is $p_{1}=3$, and the protection level for set $S_{2}$ is $p_{2}=12$. $\left(S_{3}\right.$ has a protection level equal to capacity, $p_{3}=C$ by definition.) Assuming the capacity is $C=20$, the booking limit for $M$ is $b_{3}=C-p_{2}=20-12=8$; the booking limit for $Q$ is $b_{2}=C-p_{1}=20-3=17$; and the booking limit for $Y$ is $b_{1}=C-p_{0}=C-0=C$.

It's worth emphasizing at this point that the sequence of nondominated sets, whether they are nested or not, and their nesting order are all a function of the particular choice model and the revenue values. Thus far, we have assumed these parameters are the same for all times $t$. But nothing in the analysis prevents us from having a different choice model and different fares at each time $t$. In this time-varying case, however, the nondominated sets could change with time, and if they are nested, the nesting order could change as well. This would make the implementation more complex. However, at each point in time $t$, the basic structure of the optimal policy is the one described above. 


\begin{tabular}{r|r|rrr|r}
\multicolumn{7}{|c}{$R_{k}-Q_{k} \Delta V_{t-1}(x)$} \\
$x$ & $\Delta V_{t-1}(x)$ & $k=1$ & $k=2$ & $k=3$ & $k_{t}^{*}(x)$ \\
\hline 1 & 780.00 & 6.00 & -159.00 & -275.00 & 1 \\
2 & 624.00 & 52.80 & -34.20 & -119.00 & 1 \\
3 & 520.00 & 84.00 & 49.00 & -15.00 & 1 \\
4 & 445.71 & 106.29 & 108.43 & 59.29 & 2 \\
5 & 390.00 & 123.00 & 153.00 & 115.00 & 2 \\
6 & 346.67 & 136.00 & 187.67 & 158.33 & 2 \\
7 & 312.00 & 146.40 & 215.40 & 193.00 & 2 \\
8 & 283.64 & 154.91 & 238.09 & 221.36 & 2 \\
9 & 260.00 & 162.00 & 257.00 & 245.00 & 2 \\
10 & 240.00 & 168.00 & 273.00 & 265.00 & 2 \\
11 & 222.86 & 173.14 & 286.71 & 282.14 & 2 \\
12 & 208.00 & 177.60 & 298.60 & 297.00 & 2 \\
13 & 195.00 & 181.50 & 309.00 & 310.00 & 3 \\
14 & 183.53 & 184.94 & 318.18 & 321.47 & 3 \\
15 & 173.33 & 188.00 & 326.33 & 331.67 & 3 \\
16 & 164.21 & 190.74 & 333.63 & 340.79 & 3 \\
17 & 156.00 & 193.20 & 340.20 & 349.00 & 3 \\
18 & 148.57 & 195.43 & 346.14 & 356.43 & 3 \\
19 & 141.82 & 197.45 & 351.55 & 363.18 & 3 \\
20 & 135.65 & 199.30 & 356.48 & 369.35 & 3
\end{tabular}

Table 4: Illustration of nested policy for Example 1

\subsection{Nesting by fare order}

Fares provide a natural nesting ordering, and traditionally this is how most revenue management systems have been conceived and implemented. From a practical standpoint, therefore, it is important to understand when a particular choice model leads to nesting by fare order. Yet Example 1 makes clear that nesting by fare order need not be the optimal policy in general; some choice models have this property, others do not. Next, we derive conditions that guarantee a given model will always have this property.

Recall the set of products $N=\{1, \ldots, n\}$ is assumed to be indexed so that $r_{1} \geq r_{2} \geq$ $\ldots r_{n} \geq 0$. We will say a set is complete if it is of the form $A_{k}=\{1, \ldots, k\}$ for some $k$ and incomplete otherwise.

As an aside, note that completeness of the nondominated sets is an important property if one wants to use a bid price control. In bid price controls, we set a threshold price that can depend on $t$ and $x$, denoted $\pi_{t}(x)$, such that $j$ is opened for sale if and only if $r_{j} \geq \pi_{t}(x)$. Indeed, the following proposition is easily seen to hold: 
Proposition 4 A bid price policy is optimal if and only if the nondominated sets $S_{1}, . ., S_{m}$ are complete.

In other words, if the nondominated sets are complete, then a bid price control can be used to implement it because a threshold price can be used to separate the fares in $A_{k}=\{1, \ldots, k\}$ from those in $N-A_{k}=\{k+1, \ldots, n\}$. If some nondominated sets are incomplete, this simple procedure fails.

We next examine the implications of complete sets for nesting by fare order. Recall that Proposition 1 (slightly rearranged) states that for any value $v>0$, nondominated sets are the only solutions to

$$
\max _{S \subset N} \sum_{j=1}^{n}\left(r_{j}-v\right) P_{j}(S) .
$$

Let $x_{j}=r_{j}-v$ and note that $x_{1} \geq x_{2} \geq \ldots x_{n} \geq 0$ if $r_{1} \geq r_{2} \geq \ldots r_{n} \geq 0$. The following is a definition of the nested-by-fare-order property:

Definition $4 A$ choice model $P_{j}(S), j=1, \ldots, n, S \subseteq N$ has the nesting-by-fare-order property if it satisfies the two conditions:

i) The probability of purchase, $Q(S)=\sum_{j \in S} P_{j}(S)$, is increasing in $S$, i.e. $Q(T) \geq Q(S)$ if $S \subseteq T$.

ii) For every set of values $x_{1} \geq x_{2} \geq \ldots \geq x_{n}$, the problem

$$
\max _{S \subset N} \sum_{j=1}^{n} x_{j} P_{j}(S)
$$

has an optimal solution that is complete.

In words, Property ii) guarantees that for any set of fare values $r_{1} \geq r_{2} \geq \ldots r_{n} \geq 0$, the nondominated sets are complete, while Property i) insures that when the complete sets are sequenced in increasing probability and revenue order, the sequence is $A_{1}, A_{2}, \ldots, A_{n}$. Thus, by Theorem 1, the optimal policy will be nested by fare order.

A choice model will fail to have the nested-by-fare-class property if there is a vector of fares $r$ that make an incomplete set one of the strongly nondominated sets. Here is a simple example where this is the case:

Example 2: Let $N=\{1,2,3\}$, and $r_{1}=\$ 410, r_{2}=\$ 110$ and $r_{3}=\$ 60$ and take $v=10$ so that $x_{1}=\$ 400, x_{2}=\$ 100$ and $x_{3}=\$ 50$. Let the choice probabilities conditioned on the choice set be given as in Table 3.2. Table 3.2 shows that the set that maximizes $\sum_{j} x_{j} P_{j}(S)$ is $\{1,3\}$, an incomplete set. Thus, the nesting-by-fare-order property does not hold for this choice model. ${ }^{1}$

\footnotetext{
${ }^{1}$ This specific choice system may appear odd, but it can arise if there is a mixture of unidentifiable passenger types. For instance, suppose customers are Type A or B with probability 0.5. A customer of type
} 


\begin{tabular}{l|l|r} 
Choice Set & Probability of purchase & $\sum_{j=1}^{n} x_{j} P_{j}(S)$ \\
\hline$\{3\}$ & $\{0.5\}$ & 25 \\
$\{2\}$ & $\{0.5\}$ & 50 \\
$\{1\}$ & $\{0.5\}$ & 200 \\
$\{1,2\}$ & $\{0.0,0.5\}$ & 50 \\
$\{2,3\}$ & $\{0.5,0.5\}$ & 75 \\
$\{1,3\}$ & $\{0.5,0.5\}$ & 225 \\
$\{1,2,3\}$ & $\{0.0,0.5,0.5\}$ & 75
\end{tabular}

Table 5: Choice probabilities and objective function for Example 2

The next theorem gives a characterization of choice models for which the nested-byfare-order property holds:

Theorem 2 A choice model has the nested-by-fare-order property iff i) The probability of purchase, $Q(S)$, is increasing, and ii) for every incomplete set $T$ there exist a set of convex weights $\alpha_{j}, j=1, \ldots, n$, satisfying $\alpha_{j} \geq 0$ and $\sum_{j=1}^{n} \alpha_{j}=1$, such that the probabilities defined by

$$
\bar{P}_{j}(\alpha)=\sum_{k=1}^{n} \alpha_{k} P_{j}\left(S_{k}\right), \quad j=1, \ldots, n
$$

satisfy

$$
\sum_{j=1}^{i} \bar{P}_{j}(\alpha) \geq \sum_{j=1}^{i} P_{j}(T), \quad i=1, \ldots, n-1
$$

and

$$
\sum_{j=1}^{n} \bar{P}_{j}(\alpha)=\sum_{j=1}^{n} P_{j}(T)
$$

Readers familiar with the theory of majorization (see Marshall and Olkin [29]) will note that the above conditions are equivalent to saying that the vector $\left(\bar{P}_{1}(\alpha), \ldots, \bar{P}_{n}(\alpha)\right)$ majorizes the vector $\left(P_{1}(T), \ldots, P_{n}(T)\right)$. In words, it says that the nested-by-fare-order property holds if we can find a convex combination of complete sets that has the same probability of purchase as the incomplete set $T$ (i.e. $\sum_{j=1}^{n} \bar{P}_{j}(\alpha)=\sum_{j=1}^{n} P_{j}(T)=Q(T)$ ). Yet the convex combination produces at least as high a probability of purchasing each of the nested sets $\{1\},\{1,2\}, \ldots,\{1,2, . ., n-1\}$. This later property is sufficient to ensure that the expected revenue is at least as large since it implies that for all $i=1, \ldots, n$

$$
P_{i}(T)-\bar{P}_{i}(\alpha) \leq \sum_{j=1}^{i-1} \bar{P}_{j}(\alpha)-\sum_{j=1}^{i-1} P_{j}(T) .
$$

$\mathrm{A}$ is eligible for fare products 1 and 2 only and customer type B can afford only 3. Customer A will buy fare product 2 if both 1 and 2 are available and fare product 1 if only fare product 1 is available. Then the resulting mixture probability will be as given in Table 3.2 . 
So if we use $T$ rather than the convex combination of complete set, then any increase in the probability of selling product $i$ (the left hand side) is always less than the loss in probability of selling one of the higher revenue products $1, \ldots, i-1$ (the right hand side). Stated another way, if we sell less of product $i$ using the convex combination, it is only because we are selling more of products with even higher revenues. Thus, the convex combination produces at least as much expected revenue and for the same probability of purchase.

We next give the proof:

$\underline{\text { Proof }}$

For Part i), the increasing property of the probability model is a requirement from the definition of the nested-by-fare-order property.

For Part ii), let $T$ be an incomplete set. If the probability model has the nested-by-fareorder property, then there does not exist a set of values $x_{1} \geq x_{2} \geq \ldots \geq x_{n}$ such that $T$ is the unique solution to (9). Thus, the following linear system of inequalities (in the variables $x$ and the scalar $u$ ) has no solution

$$
\begin{aligned}
& u-\sum_{k=1}^{n} P_{j}(T) x_{j}<0 \\
& \alpha_{k}: \quad u-\sum_{k=1}^{n} P_{j}\left(A_{k}\right) x_{j} \geq 0, \quad k=1, \ldots, n \\
& z_{j}: \quad x_{j}-x_{j+1} \geq 0, \quad j=1, \ldots, n-1 .
\end{aligned}
$$

But by Farka's lemma, the system (11) has no solution if and only if the dual system (in variables $\alpha_{k}, k=1, \ldots, n$ and $z_{j}, j=0,1, \ldots, n$ is solvable:

$$
\begin{gathered}
\sum_{k=1}^{n} \alpha_{k} P_{j}\left(A_{k}\right)-z_{j}+z_{j-1}=P_{j}(T), \quad j=1, \ldots, n \\
\sum_{k=1}^{n} \alpha_{k}=1 \\
\alpha \geq 0 \quad, \quad z \geq 0 .
\end{gathered}
$$

where we define $z_{0}=0$ and $z_{n}=0$ to simplify the notation.

We can next eliminate the $z$ variables. Note for $j=1$ from the first equation above together with $z_{1} \geq 0$ we have $\sum_{k=1}^{n} \alpha_{k} P_{1}\left(A_{k}\right)-P_{1}(T)=z_{1} \geq 0$, which implies

$$
\sum_{k=1}^{n} \alpha_{k} P_{1}\left(A_{k}\right) \geq P_{1}(T)
$$

For $j=2, \ldots, n-1$ using $z_{j} \geq 0$ we obtain $\sum_{k=1}^{n} \alpha_{k} P_{j}\left(A_{k}\right)-P_{j}(T)+z_{j-1}=z_{j} \geq 0$. Substituting for $z_{j-1}$ recursively we obtain

$$
\sum_{i=1}^{j} \sum_{k=1}^{n} \alpha_{k} P_{i}\left(A_{k}\right) \geq \sum_{i=1}^{j} P_{i}(T) .
$$


Similarly, for $j=n$ since $z_{n}=0$ by definition this same step gives $\sum_{i=1}^{n} \sum_{k=1}^{n} \alpha_{k} P_{i}\left(A_{k}\right)=$ $\sum_{i=1}^{n} P_{i}(T)$. Thus, there exists weights $\alpha$ satisfying the conditions of the theorem if and only if the probability model has the nested-by-fare-order property.

Although verification of the conditions of the theorem in general involves testing all incomplete subsets $T$, verifying it for a specific model or functional form is often simpler. Indeed, below we apply the basic theory to two special cases - the traditional independent demand model and the multinomial logit model - and show that the optimal policy in each case is nested by fare order.

Finally note that while Theorem 2 is stated in terms of the family of complete sets $A_{1}, A_{2}, \ldots, A_{n}$, the proof does not rely on the fact that the sets are complete. Indeed, the theorem holds for any specified family of sets. In particular, if one conjectures that the nondominated sets always comes from a particular family, one can use Theorem 2 to try to verify this conjecture.

\subsection{Independent demand model}

In the independent demand model, customers arrive at random and only want one of $n$ available fare products. This is the traditional yield management model of Lee and Hersh [26] and corresponds to the following choice model in our formulation:

$$
P_{j}(S)=\left\{\begin{array}{ll}
q_{j} & j \in S \\
0 & \text { otherwise }
\end{array} \quad \forall S \subseteq N .\right.
$$

That is, an arriving customer chooses Class $j$ with probability $q_{j}$ independent of the choice set offered and if $j$ is not offered, the customer does not purchase. We will show:

Proposition 5 For the independent model, the only nondominated sets are the complete sets $A_{k}, k=1, \ldots, n$. Moreover, the optimal policy is a nested allocation policy where the nesting is by fare order.

$\underline{\text { Proof }}$

First, it is clear that the independent demand model is increasing since $Q(S)=\sum_{i \in S} q_{i}$. Verifying Condition ii) of Theorem 2 is also quite easy: let $T$ be an incomplete set and define the index $k^{*}$ by

$$
\sum_{i=1}^{k^{*}} q_{i} \leq \sum_{i \in T} q_{i}<\sum_{i=1}^{k^{*}+1} q_{i} .
$$

Note that $k^{*}$ is less than the largest index in $T$; that is, $\max \{j: j \in T\}>k^{*}$ (otherwise, $T=A_{k^{*}}$ and it is complete). Next, define $\lambda$ by

$$
\lambda \sum_{i=1}^{k^{*}} q_{i}+(1-\lambda) \sum_{i=1}^{k^{*}+1} q_{i}=\sum_{i \in T} q_{i}
$$


and define the convex weights

$$
\alpha_{k}= \begin{cases}\lambda & k=k^{*} \\ 1-\lambda & k=k^{*}+1 \\ 0 & \text { otherwise }\end{cases}
$$

Using these weights we have that for $j \leq k^{*}$

$$
\begin{aligned}
\bar{P}_{j}(\alpha) & =\lambda P_{j}\left(A_{k^{*}}\right)+(1-\lambda) P_{j}\left(A_{k^{*}+1}\right) \\
& =\lambda q_{j}+(1-\lambda) q_{j} \\
& =q_{j} \geq P_{j}(T) .
\end{aligned}
$$

Summing for all values $i \leq j$ we obtain that for $j \leq k^{*}, \sum_{i=1}^{j} \bar{P}_{j}(\alpha) \geq \sum_{i=1}^{j} P_{j}(T)$ as required by Theorem 6 . For $j>k^{*}$ we have by (12)

$$
\sum_{i=1}^{j} \bar{P}_{i}(\alpha)=\sum_{i \in T} q_{i} \geq \sum_{i=1}^{j} P_{i}(T)
$$

with equality holding throughout when $j=n$. This completes the proof of Condition ii) of Theorem 2.

Note that one can also show that the complete sets $A_{k}$ are the only nondominated sets directly without using Theorem 2 . Indeed, by Proposition 1 , the only nondominated sets are those that maximize $R(S)-v Q(S)$ over $S \subseteq N$ for some nonnegative $v$. For the independent model,

$$
R(S)=\sum_{j \in S} r_{j} q_{j} \quad \text { and } \quad Q(S)=\sum_{j \in S} q_{j}
$$

so this optimization can be written as

$$
\begin{aligned}
& \max \sum_{j=1}^{n}\left(r_{j}-v\right) q_{j} z_{j} \\
& z_{j} \in\{0,1\}, \quad j=1, \ldots, n .
\end{aligned}
$$

An optimal solution to this problem is easily seen to be

$$
z_{j}^{*}= \begin{cases}1 & r_{j}>v \\ 0 & \text { otherwise }\end{cases}
$$

Because $r_{1} \geq r_{2} \ldots \geq r_{n}$, this solution corresponds to the set $A_{k}=\{1,2, \ldots, k\}$ where $k$ is the largest value $j$ such that $r_{j}>v$.

As a result of the nested-by-fare-order property, we recover the Lee and Hersh [26] result as we should. But more importantly we see exactly why the independent choice model leads to an optimal policy of this form. 


\subsection{Multinomial logit (MNL) choice model}

We now turn to a slightly more complex choice model - the multinomial logit (MNL) model. The MNL is used widely in travel demand forecasting and marketing (see Ben-Akiva and Lerman [10].) In the MNL, consumers are utility maximizers and the utility of each choice is a random variable. Formally, the utility of each alternative $j$ is assumed to be of the form

$$
U_{j}=u_{j}+\xi_{j}
$$

where $u_{j}$ is the mean utility of choice $j$ and $\xi_{j}$ is an i.i.d., Gumbel random noise term with mean zero and scale parameter one for all $j$. (The Gumbel, or double-exponential, distribution with zero mean and scale parameter is $F(x)=\exp \left(-e^{-(x+\gamma)}\right), x \geq 0$ where $\gamma \approx 0.577$ is Euler's constant.) Because utility is an ordinal measure, the assumption of zero mean and a scale parameter of one are without loss of generality (see Ben-Akiva and Lerman [10]). Similarly, there is a no-purchase option where the no-purchase utility is assumed to be

$$
U_{0}=u_{0}+\xi_{0}
$$

where and $\xi_{0}$ is also Gumbel with mean zero and scale parameter one. Again, since utility is ordinal, without loss of generality we can assume $u_{0}=0$.

Under this utility model, one can show (See Ben-Akiva and Lerman [10] for a derivation.) that the choice probabilities are given by

$$
P_{j}(S)=\frac{e^{u_{j}}}{\sum_{i \in S} e^{u_{i}}+e^{u_{o}}}, \quad j \in S \text { or } j=0 .
$$

and zero otherwise. For notational convenience, we define "weights" $w_{j}=e^{u_{j}}, j=0,1 \ldots, n$, so that the choice probabilities can be expressed as

$$
P_{j}(S)=\frac{w_{j}}{\sum_{i \in S} w_{i}+1}, j \in S \text { or } j=0 .
$$

That is, the probability of choosing $j$ is its "weight" $w_{j}$ divided by the weights of all other choices in $S$. (Recall, $u_{0}=0$ so $w_{0}=1$.) Note that since $e^{x}$ is monotone increasing in $x$, higher values of $u_{j}$ imply higher values of $w_{j}$.

We next show that for this choice model, the optimal policy is again nested by fare order:

Proposition 6 For the MNL choice model, the only nondominated sets are the complete sets $A_{k}, k=1, \ldots, n$. Moreover, the optimal policy is a nested allocation policy where the nesting is by fare order.

Before proving the result, we need a simple lemma: 
Lemma 5 Consider the functions $f(y)=\frac{y}{y+1}$ and $g(y)=\frac{1}{y+1}$. Suppose $y_{1} \leq y_{2} \leq y_{3}$ and $\lambda$ are nonnegative numbers satisfying $\lambda f\left(y_{1}\right)+(1-\lambda) f\left(y_{3}\right)=f\left(y_{2}\right)$. Then $\lambda g\left(y_{1}\right)+(1-$ $\lambda) g\left(y_{3}\right)=g\left(y_{2}\right)$ as well.

The proof follows easily from the fact that $g(y)=1-f(y)$.

We now turn to the proof of Theorem 6 :

$\underline{\text { Proof }}$

First, is clear from the definition of the MNL probabilities (14) that the total probability of purchase $Q(S)=\sum_{j \in S} P_{j}(S)$ is increasing in $S$.

To show the second set of conditions of Theorem 2, let $T$ be an incomplete set and again define the index $k^{*}$ by

$$
\sum_{i=1}^{k^{*}} w_{i} \leq \sum_{i \in T} w_{i}<\sum_{i=1}^{k^{*}+1} w_{i}
$$

Note that $k^{*}$ is less than the largest index in $T$; that is, $\max \{j: j \in T\}>k^{*}$ as before. Next, define $\lambda$ by

$$
\lambda \frac{\sum_{i=1}^{k^{*}} w_{i}}{\sum_{i=1}^{k^{*}} w_{i}+1}+(1-\lambda) \frac{\sum_{i=1}^{k^{*}+1} w_{i}}{\sum_{i=1}^{k^{*}+1} w_{i}+1}=\frac{\sum_{i \in T} w_{i}}{\sum_{i \in T} w_{i}+1}
$$

and note by our Lemma 5 that

$$
\lambda \frac{1}{\sum_{i=1}^{k^{*}} w_{i}+1}+(1-\lambda) \frac{1}{\sum_{i=1}^{k^{*}+1} w_{i}+1}=\frac{1}{\sum_{i \in T} w_{i}+1}
$$

as well. Also define the convex weights

$$
\alpha_{k}= \begin{cases}\lambda & k=k^{*} \\ 1-\lambda & k=k^{*}+1 \\ 0 & \text { otherwise }\end{cases}
$$

Using these weights and the MNL probabilities (14), we have that for $j \leq k^{*}$

$$
\begin{aligned}
\bar{P}_{j}(\alpha) & =\lambda P_{j}\left(A_{k^{*}}\right)+(1-\lambda) P_{j}\left(A_{k^{*}+1}\right) \\
& =\lambda \frac{w_{j}}{\sum_{i=1}^{k^{*}} w_{i}+1}+(1-\lambda) \frac{w_{j}}{\sum_{i=1}^{k^{*}+1} w_{i}+1} \\
& =\frac{w_{j}}{\sum_{i \in T} w_{i}+1} \\
& \geq P_{j}(T),
\end{aligned}
$$

where the second equality follows from (16) and the last inequality follows from the fact that $P_{j}(T)=w_{j} /\left(\sum_{i \in T} w_{i}+1\right)$ if $j \in T$ and $P_{j}(T)=0$ otherwise. Summing for all values 
$i \leq j$ we obtain that for $j \leq k^{*}, \sum_{i=1}^{j} \bar{P}_{j}(\alpha) \geq \sum_{i=1}^{j} P_{j}(T)$ as required by Theorem 2 . For $j>k^{*}$ we have by $(15)$

$$
\begin{aligned}
\sum_{i=1}^{j} \bar{P}_{i}(\alpha) & =\sum_{i=1}^{k^{*}}\left(\lambda \frac{w_{i}}{\sum_{l=1}^{k^{*}} w_{l}+1}+(1-\lambda) \frac{w_{i}}{\sum_{l=1}^{k^{*}+1} w_{l}+1}\right)+(1-\lambda) \frac{w_{k^{*}+1}}{\sum_{l=1}^{k^{*}+1} w_{l}+1} \\
& =\lambda \sum_{i=1}^{k^{*}} \frac{w_{i}}{\sum_{l=1}^{k^{*}} w_{l}+1}+(1-\lambda) \sum_{i=1}^{k^{*}+1} \frac{w_{i}}{\sum_{l=1}^{k^{*}+1} w_{l}+1} \\
& =\frac{\sum_{i \in T} w_{i}}{\sum_{i \in T} w_{i}+1} \\
& \geq \sum_{i=1}^{j} P_{i}(T)
\end{aligned}
$$

And finally, evaluating the above for $j=n$ shows that $\sum_{i=1}^{n} \bar{P}_{i}(\alpha)=\sum_{i=1}^{n} P_{i}(T)$, which completes the proof.

The above proof shows that a convex combination of the two complete sets $A_{k^{*}}$ and $A_{k^{*}+1}$ always dominates the incomplete set $T$ for any give vector of fares $r$. Thus, for the MNL model a nested allocation policy where the nesting is by fare order is again optimal. However, the nested allocation are not necessarily the same as in the independent model.

Indeed, by reexamining the optimality condition one can gain some additional insight into the different allocations produced by the MNL model. From Proposition 6 , it is not hard to show that it is optimal to open fare $k+1$ if and only if

$$
r_{k+1}-\Delta V_{t-1}(x) \geq \sum_{j=1}^{k} P_{j}\left(A_{k}\right)\left(r_{j}-\Delta V_{t-1}(x)\right) .
$$

This expression is intuitive: The left hand side is the "net gain" from selling class $k+1$; that is, the revenue we get from class $k+1$ minus the opportunity cost, $\Delta V_{t-1}(x)$, of using a unit of capacity. The right hand side is the net gain from offering only classes $A_{k}=\{1, \ldots, k\}$ (e.g. the sum over all classes in $A_{k}$ of the probability that a customer chooses $j$ from $A_{k}$ times the net gain from selling $j$.). The condition (17) simply says that if the net gain from selling $k+1$ is more profitable than the gamble of offering only $A_{k}$, then it pays to open $k+1$; else, $k+1$ should be closed.

The expression (17) should be compared to the optimality condition for independent demand model; namely, it is optimal to open class $k+1$ if and only if

$$
r_{k+1}-\Delta V_{t-1}(x) \geq 0
$$

Note the right hand side above is zero while the right hand side of (17) is positive. This happens because in the independent demand model, if we close class $k+1$, we lose all demand for that class. Therefore it is optimal to accept fare $k+1$ whenever $r_{k+1}$ exceeds 
the opportunity cost $\Delta V_{t-1}(x)$. However, in the MNL model, if we close class $k+1$ customers chose from among the other classes that are offered (e.g. from $A_{k}$ ). Hence, the threshold on the right hand side of (17) is greater than zero. This difference reflects the fact that customers may "buy-up" to a higher class.

\section{Estimation}

We next consider the problem of estimating choice model parameters from historical data. We will use the MNL model for illustration. While we focus on the MNL case, the basic estimation ideas developed below work with essentially any choice model for which maximum likelihood methods can be applied.

To estimate the mean utility in the MNL, it is common to model utility as a linear function of several known attributes (e.g., price, indicator variables for product restrictions, etc.), much as one would do in a linear regression model. Thus, $u_{j}=\beta^{T} x_{j}$ where $x_{j}$ is a vector of known attributes of choice $i$ and $\beta$ is a vector of weights on these variables. The weights $\beta$ are to be estimated from historical data.

Estimation of the MNL model given a complete set of choice data is a well-studied problem. In particular, the maximum likelihood estimate (MLE) has good computational properties (Its log is jointly concave in most cases; See McFadden [30].), and the method has proved robust in practice. (See Ben-Akiva and Lerman [10] for further discussion and case examples.)

In our case, we have an arrival probability as well as choice parameters to estimate. However, given complete observations, estimation for our model is only a slight modification of the MNL case. In particular, let $D$ denote a set of intervals, indexed by $t$, in which independent arrival events and choice decisions have been observed. The set $D$ could combine intervals from many flight departures and, deviating somewhat from our notational convention thus far, $t$ here does not necessarily represent the time remaining for a particular flight. For each period $t \in D$, let

$$
a_{t}= \begin{cases}1 & \text { if customer arrives in period } t \\ 0 & \text { otherwise }\end{cases}
$$

Let $A$ denotes the set of periods $t$ with arrivals $\left(a_{t}=1\right)$ and $\bar{A}=D-A$ denote the periods with no arrivals. If $t \in A$, let $j(t)$ denote the choice made by the arriving customer. (For

$t \in \bar{A}$ define $j(t)$ arbitrarily.) Finally, as before let $S$ denote the set of open fare products in interval $t$. The likelihood function is then

$$
\prod_{t \in D}\left[\lambda \frac{e^{\beta^{T} x_{j(t)}}}{\sum_{j \in S} e^{\beta^{T} x_{j}}+1}\right]^{a_{t}}(1-\lambda)^{\left(1-a_{t}\right)}
$$


Taking logs, we obtain the log-likelihood function

$$
\mathcal{L}=\sum_{t \in D}\left[a_{t}\left(\beta^{T} x_{j(t)}-\ln \left(\sum_{j \in S} e^{\beta^{T} x_{j}}+1\right)\right)+a_{t} \ln (\lambda)+\left(1-a_{t}\right) \ln (1-\lambda)\right] .
$$

Note that $\mathcal{L}$ is separable in $\beta$ and $\lambda$. Maximizing $\mathcal{L}$ with respect to $\lambda$, we obtain the estimate

$$
\hat{\lambda}=\frac{1}{|D|} \sum_{t \in D} a_{t}=\frac{|A|}{|D|}
$$

where $|D|$ (resp. $|A|$ ) denotes the cardinality of $D$ (resp. $|A|$ ). The MLE, $\hat{\beta}$, is then determined by solving

$$
\max _{\beta} \sum_{t \in A}\left(\beta^{T} x_{j(t)}-\ln \left(\sum_{j \in S} e^{\beta^{T} x_{j}}+1\right)\right)
$$

This is simply the usual maximum likelihood problem for the MNL applied to those periods with customer arrivals. Combining these two estimates gives the MLE for the MNL choice model with complete data.

As mentioned, the difficulty with this approach in practice is that one rarely observes all arrivals. Typically, only purchase transaction data are available. Thus, it is impossible to distinguish a period without an arrival, from a period in which there was an arrival but the arriving customer did not purchase. With this incompleteness in the data, the above MLE procedure cannot be used. One could always try to write down the likelihood function with incomplete data, but typically the function becomes very complex and difficult to maximize.

To overcome this problem, we propose using the expectation-maximization (EM) method of Dempster et al. [17]. The method works by starting with arbitrary initial estimates, $\hat{\beta}$ and $\hat{\lambda}$. These estimates are then used to compute the conditional expected value of $\mathcal{L}$ : $E[\mathcal{L} \mid \hat{\beta}, \hat{\lambda}]$ (the expectation step). The resulting expected log-likelihood function is then maximized to generate new estimates $\hat{\beta}$ and $\hat{\lambda}$ (the maximization step) and the procedure is repeated until it converges. While technical convergence problems can arise, in practice the EM method is a robust and efficient way to compute maximum likelihood estimates for incomplete data. (See McLachlan and Krishnan [33] for a comprehensive reference on the EM method.) It has also been used in other yield management contexts, in particular by McGill [31] to estimate multi-variate normal demand data with censoring.

To apply the EM method in our case, let $P$ denote the set of periods in which customers purchase and $\bar{P}=D-P$ denote period in which there are no purchase transactions. We can then write the complete log-likelihood function as

$$
\begin{aligned}
\mathcal{L}= & \sum_{t \in P}\left[\ln (\lambda)+\beta^{T} x_{j(t)}-\ln \left(\sum_{j \in S} e^{\beta^{T} x_{j}}+1\right)\right] \\
& +\sum_{t \in \bar{P}}\left[a_{t}\left(\ln (\lambda)-\ln \left(\sum_{j \in S} e^{\beta^{T} x_{j}}+1\right)\right)+\left(1-a_{t}\right) \ln (1-\lambda)\right] .
\end{aligned}
$$


The unknown data are the values $a_{t}, t \in \bar{P}$ in the second sum. However, given estimates $\hat{\beta}$ and $\hat{\lambda}$, we can determine their expected values (denoted $\hat{a}_{t}$ ) easily via Bayes's rule:

$$
\begin{aligned}
\hat{a}_{t} \doteq E\left[a_{t} \mid t \in \bar{P}, \hat{\beta}, \hat{\lambda}\right] & =P\left(a_{t}=1 \mid t \in \bar{P}, \hat{\beta}, \hat{\lambda}\right) \\
& =\frac{P\left(t \in \bar{P} \mid a_{t}=1, \hat{\beta}, \hat{\lambda}\right) P\left(a_{t}=1 \mid \hat{\beta}, \hat{\lambda}\right)}{P(t \in \bar{P} \mid \hat{\beta}, \hat{\lambda})} \\
& =\frac{\hat{\lambda} P_{0}(S \mid \hat{\beta})}{\hat{\lambda} P_{0}(S \mid \hat{\beta})+(1-\hat{\lambda})},
\end{aligned}
$$

where

$$
P_{0}(S \mid \hat{\beta})=\frac{1}{\sum_{j \in S} e^{\hat{\beta}^{T} x_{j}}+1}
$$

is the no-purchase probability for observation $t$ given $\hat{\beta}$.

Substituting $\hat{a}_{t}$ into (20) we obtain the expected log-likelihood for the incomplete data

$$
\begin{aligned}
& E[\mathcal{L} \mid \hat{\beta}, \hat{\lambda}]=\sum_{t \in P}\left[\beta^{T} x_{j(t)}-\ln \left(\sum_{j \in S} e^{\beta^{T} x_{j}}+1\right)\right]-\sum_{t \in \bar{P}} \hat{a}_{t} \ln \left(\sum_{j \in S} e^{\beta^{T} x_{j}}+1\right) \\
& +\sum_{t \in P} \ln (\lambda)+\sum_{t \in \bar{P}}\left(\hat{a}_{t} \ln (\lambda)+\left(1-\hat{a}_{t}\right) \ln (1-\lambda)\right) .
\end{aligned}
$$

As in the case of the complete log-likelihood function, this function is separable in $\beta$ and $\lambda$. Maximizing with respect to $\lambda$ we obtain the updated estimate

$$
\lambda^{*}=\frac{|P|+\sum_{t \in \bar{P}} \hat{a}_{t} .}{|P|+|\bar{P}|} .
$$

This is intuitive; our estimate of lambda is the number of observed arrivals, $|P|$, plus the estimated number of arrivals from unobservable periods, $\sum_{t \in \bar{P}} \hat{a}_{t}$, divided by the total number of periods $|P|+|\bar{P}|=|D|$. We can then maximize the first two sums in (22) to obtain the updated estimate $\beta^{*}$. Note that this expression is of the same functional form as the complete data case (19). The entire procedure is then repeated.

Summarizing the algorithm:

Step 0: Initialize $\hat{\beta}$ and $\hat{\lambda}$.

Step 1: Expectation step

- For $t \in \bar{P}$, use the current estimates $\hat{\beta}$ and $\hat{\lambda}$ to compute $\hat{a}_{t}$ from (21).

Step 2: Maximization step

- Compute $\lambda^{*}$ using (23). 
- Compute $\beta^{*}$ by solving

$$
\max _{\beta}\left\{\sum_{t \in P}\left(\beta^{T} x_{j(t)}-\ln \left(\sum_{j \in S} e^{\beta^{T} x_{j}}+1\right)\right)-\sum_{t \in \bar{P}} \hat{a}_{t} \ln \left(\sum_{j \in S} e^{\beta^{T} x_{j}}+1\right)\right\}
$$

Step 3: Convergence test

$$
\begin{aligned}
& \text { - IF }\left\|(\hat{\lambda}, \hat{\beta})-\left(\lambda^{*}, \beta^{*}\right)\right\|<\epsilon, \text { THEN STOP; } \\
& \text { - ELSE } \hat{\lambda} \leftarrow \lambda^{*}, \hat{\beta} \leftarrow \beta^{*} \text { and GOTO Step } 1 .
\end{aligned}
$$

Since the expected log-likelihood, $E[\mathcal{L} \mid \hat{\beta}, \hat{\lambda}]$, given in $(22)$ is continuous in both $(\beta, \lambda)$ and $(\hat{\beta}, \hat{\lambda})$, a result by $\mathrm{Wu}[46]$ shows that if the sequence of estimates converges, the resulting value will be a stationary point of the incomplete log-likelihood function. Whether the sequence diverges - or converges to something other than the global maximum - is more difficult to determine. In practice, the method has proved to be very robust in other contexts and this has been our experience in simulated experiments for our problem. (See McLachlan and Krishnan [33] for further discussion of convergence properties of the EM algorithm.)

One interesting fact is that there can be multiple pairs $(\beta, \lambda)$ that produce the same probabilities of sales. In this case, the EM and MNL estimates will only find one such pair. To take a trivial case, suppose there is only $n=1$ fare product and that $x_{1}$ and $\beta$ are scalars. The probability that we observe a sale if this fare product is open is then

$$
p=\lambda \frac{e^{\beta x_{1}}}{e^{\beta x_{1}}+1}
$$

It is clear that there are a continuum of values $(\beta, \lambda)$ that will produce the same value $p$. However, the maximum likelihood estimate will identify only one such pair. This difficulty is not a fault of the EM or MNL method per se; it is really a reflection of the fact that as in this simple example - there may be more than one probability model that produces the same purchase probabilities. In such cases, it is simply not possible to uniquely identify the model from observed data. In such cases, there is in effect a degree of freedom that we cannot resolve.

\section{$5 \quad$ Numerical Example}

In this section we provide results of a small simulation study to compare our choice-based method to a traditional single-leg method using the MNL model as an example. Both the estimation and optimization methods were tested. We do not address the validity of the MNL model itself, but since our approach is quite general and a wide range of choice models could be used, validation of an appropriate model is something that must be done on a case-by-case basis. The MNL example used here simply serves to illustrate the basic behavior of the optimal policy relative to more traditional, heuristic approaches. 
The traditional model we tested against was Belobaba's EMSR-b heuristic with buyup [8]. This is a variation of one of the most common seat protection heuristics used in practice. We first describe the EMSR-b heuristic and the heuristic modification to account for buy-up.

EMSR-b is a fixed protection level policy, which sets a static set of protection levels for fare products 1 through $n-1$ given by the vector $x=\left(x_{1}, \ldots, x_{n-1}\right)$, where $x_{1} \leq x_{2} \leq$ $\ldots \leq x_{n-1}$. (There is no protection level for the lowest fare class, $n$.) Protection levels are nested in the sense that $x_{j}$ represents the number of inventory units to reserve (protect) for all of fare products $1,2, \ldots, j$. Reservations for fare product $j+1$ are accepted if and only if the number of inventory units remaining is strictly greater than the protection limit $x_{j}$. (Such policies are optimal when low fare products book strictly before higher fare products and fare product demands are mutually independent [13].)

EMSR-b sets the protection levels $x_{j}$ as follows: Given estimates of the means, $\hat{\mu}_{j}$, and standard deviations, $\hat{\sigma}_{j}$, for each fare product $j$, the EMSR-b heuristic sets $x_{j}$ so that

$$
r_{j+1}=\bar{r}_{j} P\left(\bar{X}_{j}>x_{j}\right),
$$

where $\bar{X}_{j}$ is a normal random variable with mean $\sum_{i=1}^{j} \hat{\mu}_{i}$ and variance $\sum_{i=1}^{j} \hat{\sigma}_{i}^{2}$, and $\bar{r}_{j}$ is a weighted average revenue, given by

$$
\bar{r}_{j}=\frac{\sum_{i=1}^{j} r_{i} \hat{\mu}_{i}}{\sum_{i=1}^{j} \hat{\mu}_{i}} .
$$

The idea behind this approximation is to reduce the complexity of the fully nested problem by aggregating fare products $1,2, . ., j$ into a single fare product. Then, one treats the problem as a simple, two-fare-class problem and applies Littlewood's rule [28].

The variation of EMSR-b with buy-up factors proposed by Belobaba [8] is as follows: Consider the simple two-class static model. Littlewoods rule (slightly restated) is to accept demand from Class 2 if and only if $r_{2}-r_{1} P\left(X_{1} \geq x\right) \geq 0$. Now suppose that there is a probability $q$ that a customer for Class 2 will buy a Class 1 fare if Class 2 is closed. The net benefit of accepting the request is still the same, but now rather than losing the request, if we reject it there is some chance the customer will sell-up to Class 1 . If so, we earn a net benefit of $r_{1}-r_{1} P\left(X_{1} \geq x\right)$ (the Class 1 revenue minus the marginal cost). Thus, it is optimal to accept Class 2 now if $r_{2}-r_{1} P\left(X_{1} \geq x\right) \geq q r_{1}\left(1-P\left(X_{1} \geq x\right)\right)$, or equivalently if

$$
r_{2} \geq(1-q) r_{1} P\left(X_{1} \geq x\right)+q r_{1} .
$$

Note that the RHS side above is strictly larger than the RHS of Littlewoods rule, which means that the above rule is more likely to reject Class 2 demand.

Using this reasoning for the 2-fare case, EMSR-b can then be extended to allow for a buy-up factor by modifying the equation for determining the protection level $x_{k}$ as follows

$$
r_{k}=\left(1-q_{k}\right) \bar{R}_{k-1} P\left(S_{k-1}>x_{k}\right)+q_{k} \hat{r}_{k-1},
$$




\begin{tabular}{l|llllllllll}
$j$ & 1 & 2 & 3 & 4 & 5 & 6 & 7 & 8 & 9 & 10 \\
\hline$r_{j}$ & 600 & 550 & 475 & 400 & 300 & 280 & 240 & 200 & 185 & 175 \\
$w_{j}^{L}$ & 0.407 & 0.438 & 0.490 & 0.549 & 0.638 & 0.657 & 0.698 & 0.741 & 0.758 & 0.769 \\
$w_{j}^{H}$ & 0.050 & 0.064 & 0.093 & 0.135 & 0.223 & 0.247 & 0.301 & 0.368 & 0.397 & 0.417
\end{tabular}

Table 6: Fares and $w_{j}$ values for numerical example

where $q_{k}$ is the probability that a customer of Class $k$ buys-up to one of the Classes $k-$ $1, k-2, \ldots, 1$ and $\hat{r}_{k-1}$ is an estimate of the average revenue received given that the customer buys-up.

Converting the choice model parameters into buy-up factors for the EMSR-b method with buy-up is relatively straightforward: It is the fraction of people who will buy any one of the higher fare products. This can be determined by summing the MNL probabilities.

To test the two methods, we simulated arrivals and applied each method to control fare product availability. The capacity was $C=185$ inventory units and there were $n=10$ fareclasses with fares as shown in Table 6. For simplicity, we assumed the random utility had only one attribute, $x$, which was simply the price. The coefficient, $\beta$, on the price attribute was taken to be either $\beta^{L}=-0.0015$ (low price sensitivity, denoted $L$ ) or $\beta^{H}=-0.005$ (high price sensitivity, denoted $H$ ). The values $w_{j}^{L}=e^{\beta^{L} x}$ and $w_{j}^{H}=e^{\beta^{H} x}$ are shown in Table 6 as well.

Arrivals over the booking period were generated by simulating a homogeneous Poisson process with mean 205. (Thus, if the booking period is broken up into intervals of size $\Delta$, then $\lambda=205 \Delta$ in the choice-based DP. We used $\Delta=1 / 410$ - or $\lambda=0.5$ - in our computations.) The choice parameter were estimated using the EM method as described in Section 4. The training set consisted of 50 simulated days during which the available classes were controlled using EMSR-b as described above. The EM method produced an estimates of $\hat{\beta}^{L}=-0.0014$ and $\hat{\beta}^{H}=-0.0048$, which are very close to the actual values of $\beta^{L}=-0.0015, \beta^{H}=-0.005$. To mimic the real-world combination of forecasting and optimization, these estimated value were used in the choice DP algorithm.

Bookings were generated for a booking period of 100 simulated days and the controls of each method were applied. The results of 15 simulated flights are shown in Table 7 . In the case where the price sensitivity is low, the choice DP has significantly higher revenues; 68,373 versus 61,049 for EMSR. This represents a $12 \%$ improvement in revenue, which is very large when compared to the typical $1-2 \%$ differences in revenues that one finds when comparing optimization methods. In the case of high price sensitivity, the revenue difference between the two methods is essentially identical within the simulation error of our test. This is not surprising since buy-up in particular can best be exploited when customers are not very price sensitive.

In terms of qualitative behavior, the choice DP frequently closes lower-fare products 


\begin{tabular}{l|ll|ll}
\multicolumn{2}{c}{} & \multicolumn{2}{c}{ Low Price Sensitivity } & \multicolumn{2}{c}{ High Price Sensitivity } \\
& Choice DP & EMSR-b Buy-Up & Choice DP & EMSR Buy-Up \\
\cline { 2 - 5 } Avg. Revenue & 68,373 & 61,049 & 36,507 & 36,414 \\
Load Factor & $74 \%$ & $90 \%$ & $72 \%$ & $77 \%$
\end{tabular}

Table 7: Simulation Results

to force consumers to buy-up to higher fares. EMSR-b, even with the buy-up heuristic, opens up more fare classes and allows customers to buy at lower prices. This difference shows up in the load factors, which are lower for the choice-based DP. While this drop in load-factors may at first be worrisome, it is not unexpected given that the choice-based DP is deliberately increasing the probability that customers will not purchase by restricting discounts. However, the revenue increases from the higher fares more than compensate for these lower volume of sales in the low-price-sensitivity case and produce essentially the same revenues in the high-price-sensitivity case. While the magnitude of these results are specific to this particular set of numbers and choice probabilities, the results do show how it may be possible to increase revenue by exploiting choice behavior.

\section{Conclusion}

Our analysis provides a quite complete characterization of optimal policies under a general choice model of demand. The fact that the optimal policy consists of selecting a set from a sequence of nondominated sets - and that the optimal set to select is further along the sequence the more capacity one has available - is strikingly simple given the prima facia complexity of the problem. Moreover, the analysis based on nondominated sets provides insight into when nested and nested-by-fare-class policies are optimal, which is useful in understanding both the traditional independent demand model, as well as new demand models such as the MNL. Finally, we provided a general method to estimate choice models when no-purchase outcomes are unobservable.

To practitioners, our choice based approach to revenue management may be a departure from current thinking, but we believe it represents the best alternative for incorporating consumer behavior into revenue management - a long-standing goal for many industry practitioners. We summarize in Table 8 how traditional revenue management functions (based on the independent demand model) map to revenue management based on consumer choice.

As for additional research, we see several topics worthy of further study. One is to try to identify other classes of probability models for which nesting - or nesting by fare order - in optimal. Another worthwhile extension would be to model choice among a set of flights. This was one of the topics investigated by Andersson at SAS [2] and Algers and Besser [1] and it would be interesting to see if the estimation and optimization methods 


\begin{tabular}{|c|c|}
\hline Traditional RM & Choice-based RM \\
\hline $\begin{array}{l}\text { Controls based on allocations } \\
\text { and bid prices }\end{array}$ & $\begin{array}{l}\text { Controls based on set of } \\
\text { alternatives to offer }\end{array}$ \\
\hline $\begin{array}{l}\text { Forecast fare product } \\
\text { demand }\end{array}$ & $\begin{array}{l}\text { Estimate consumer choice } \\
\text { model }\end{array}$ \\
\hline $\begin{array}{l}\text { Unconstrain fare product } \\
\text { demand }\end{array}$ & $\begin{array}{l}\text { Correct for unobservable } \\
\text { no-purchase events }\end{array}$ \\
\hline $\begin{array}{c}\text { Ad hoc buy-up, buy-down, recapture, } \\
\text { and spill heuristics }\end{array}$ & $\begin{array}{l}\text { Buy-up \& buy-down is implicitly considered } \\
\text { and optimized by the model }\end{array}$ \\
\hline $\begin{array}{c}\text { Fare product availability } \\
\text { optimization }\end{array}$ & $\begin{array}{c}\text { Choice-based DP } \\
\text { optimization }\end{array}$ \\
\hline
\end{tabular}

Table 8: Mapping traditional revenue management tasks to a choice-based setting

proposed here could be extended to model control of a set of related flights. Similar but even more complex would be to extend the model to networks. In both these cases, exact dynamic programming will most likely be impractical, so it would be interesting to see what approximation methods could be developed.

More generally, the choice-based approach presented here can provide a basis for other advanced practices. In particular, it can: (i) help generalize revenue management to a wide range of industries with very different, industry-specific customer purchase behavior, (ii) provide a basis for a CRM (customer relationship management) level approach to revenue management, where controls are exerted at the individual customer level, and (iii) allow one to integrate and optimize both pricing and product attribute design within revenue management.

\section{References}

[1] Algers, S. and M. Besser 2001. "Modelling choice of flight and booking class - A study using stated preference and revealed preference data," Intl. J. of Services Technology and Management, 2, 28-45.

[2] Andersson, S.E. 1998. "Passenger Choice Analysis for Seat Capacity Control: A Pilot Project in Scandinavian Airlines," Intl. Trans. Opl. Res. , 5, 471-486.

[3] Barnhart, C. and Talluri, K.T 1996. "Airline Operations Research," to appear in Systems for Civil and Environmental Engineering: An Advanced Text Book (ed. Charles ReVelle and Arthur McGarrity), John Wiley and Sons.

[4] Bellman, R. 1957. Applied Dynamic Programming, Princeton University Press, Princeton, N.J. 
[5] Belobaba, P.P. 1987. "Air Travel Demand and Airline Seat Inventory Management," Ph.D. thesis, MIT, Cambridge, Mass.

[6] Belobaba, P.P. 1987. "Airline yield Management: An Overview of Seat Inventory Control," Trans. Sci. 21, 63-73.

[7] Belobaba, P.P. 1989. "Application of a Probabilistic Decision Model to Airline Seat Inventory Control," Oper. Res. 37, 183-197.

[8] Belobaba, P.P. and L. Weatherford 1996. "," Oper. Res. 37, 183-197.

[9] Belobaba, P.P. and C. Hopperstad 1999. "Boeing/MIT Simulation Study: PODS Results Update," 1999 AGIFORS Reservations and Yield Management Study Group Symposium, April 27-30, London.

[10] Ben-Akiva, M. and S.R. Lerman, Discrete Choice Analysis, The MIT Press, Cambridge, Massachusetts.

[11] Bertsekas, D.P. 1995. Dynamic Programming and Optimal Control, Volume I., Athena Scientific, Belmont, Mass.

[12] Bitran, G. Caldentey, R. and Mondschein, S. 1998. "Coordinating Clearance Markdown Sales of Seasonal Products in Retail Chains," Oper. Res. , 46, 609-624.

[13] Brumelle, S.L., and McGill, J.I. 1993. "Airline Seat Allocation With Multiple Nested Fare Classes," Oper. Res. 41, 127-137.

[14] Brumelle, S.L., McGill, J.I., Oum, T.H., Sawaki,K., Trethwway, M.W. 1990. "Allocation of Airline Seats Between Stochastically Dependent Demand," Trans. Sci. 24, 183-192.

[15] Cooper, W. 2000. "Asymptotic Behavior of Some Revenue Management Policies," Univ. of Minnesota Working Paper, Minneapolis, Minn.

[16] Curry, R.E. 1989. Optimal Airline Seat Allocation with Fare Classes Nested by Origins and Destinations. Trans. Sci. 24, 193-204.

[17] Dempster, A.P., N.M. Laird and D.B. Rubin 1977. "Maximum Likelihood From Incomplete Data via the EM Algorithm," J. of the Royal Stat. Society, B, 39, 1-38.

[18] Dror, M., Trudeau, P. and Ladany, S.P. 1988. Network Models for Seat Allocation of Flights. Tran. Res. 22B, 239-250.

[19] Feng, Y. and Gallego, G. 1995. Optimal Stopping Times for Promotional Fares and Optimal Starting Times for End-of-Season Sales. To appear in Mgmt. Sci.

[20] Gallego, G. and G. van Ryzin 1994. "Optimal Dynamic Pricing of Inventories with Stochastic Demand Over Finite Horizons," Mgmt. Sci., 40, 999-1020 
[21] Gallego, G. and G. van Ryzin 1997. "A Multi-Product, Multi-Resource Pricing Problem and Its Applications to Network Yield Management," Oper. Res., 45, 24-41.

[22] Glover, F., Glover, R., Lorenzo, J. and McMillan, C. 1982. "The Passenger-Mix Problem in the Scheduled Airlines," Interf. 12, 73-79.

[23] Iyengar, S.S and M. Lepper (2000). "When choice is demotivating: Can one desire too much of a good thing?" J. of Personality and Social Psychology, 76, 995-1006.

[24] Kleywegt, A.J. and J.D. Papastavrou 1998. "The Dynamic and Stochastic Knapsack Problem," Operations Res., 46, 17-35.

[25] Lautenbacher, C. J. and S. J. Stidham, "The Underlying Markov Decision Process in the Single-Leg Airline Yield Management Problem," Trans. Sci., 34, 136-146.

[26] Lee, T. C., and Hersh M. 1993. A Model for Dynamic Airline Seat Inventory Control with Multiple Seat Bookings. Trans. Sci., 27, 252-265.

[27] Liang, Y. 1999. "Solution to the Continuous Time Dynamic Yield Management Model," Trans. Sci., 33, 117-123.

[28] Littlewood, K. 1972. "Forecasting and Control of Passengers," 12th AGIFORS Symposium Proceedings, 95-128.

[29] Marshall, A.W. and, I. Olkin (1979). Inequalities: Theory of Majorization and its Application, Academic Press, New York.

[30] McFadden, D. 1974. "Conditional Logit Analysis of Qualitative Choice Behavior," in Frontiers in Econometrics, P. Zarembka (ed.), Academic Press, New York, 105-142.

[31] McGill, J.I. 1995. "Censored Regression Analysis of Multiclass Demand Data Subject to Joint Capacity Constraints," Ann. Oper. Res., 60, 209-240.

[32] McGill, J. and G.J. van Ryzin 1999. "Revenue Management: Research Overview and Prospects," Trans. Sci., 33, 233-256.

[33] McLachlan, G. and T. Krishnan 1996. "The EM Algorithm and Extensions," John Wiley \& Sons, New York.

[34] Phillips, R. 1994. "State-Contingent Airline Yield Management," Presentation in Session TC33.4, INFORMS Detroit 1994.

[35] Robinson, L.W. (1991), "Optimal and Approximate Control Policies for Airline Booking with Sequential Nonmonotonic Fare Classes," Oper. Res., 43, 252-263.

[36] Simpson, R.W. (1989), "Using Network Flow Techniques to Find Shadow Prices for Market and Seat Inventory Control," MIT Flight Transportation Laboratory Memorandum M89-1, Cambridge, Massachusetts. 
[37] Smith, B., Leimkuhler, J., Darrow, R., and J. Samuels 1992. "Yield Management at American Airlines," Interfaces, 22, 8-31.

[38] Stone, R. and M. Diamond 1992. "Optimal Inventory Control for a Single Flight Leg", working paper, Northwest Airlines, Operations Research Division, Minneapolis.

[39] Subramanian, C. J. Lautenbacher, and S. J. Stidham 1999. "Yield Management with Overbooking, Cancellations and No Shows," Trans. Sci., 34, 147-167.

[40] Talluri, K.T. 1996. "Revenue Management on a Line Network," Working Paper, UPF, Barcelona, Spain.

[41] Talluri, K.T. and G.J. van Ryzin 1998. "An Analysis of Bid-Price Controls for Network Revenue Management," Mgmt. Sci., . 44, 1577-1593.

[42] Talluri, K. and G.J. van Ryzin 1999. "A Randomized Linear Programming Method for Computing Network Bid Prices," Trans. Sci.,33, 207-216.

[43] Williamson, E. L. 1988. "Comparison of Optimization Techniques for OriginDestination Seat Inventory Control," Master's thesis, Flight Transportation Laboratory, Massachusetts Institute of Technology, Cambridge, Mass.

[44] Williamson, E.L. 1992. "Airline Network Seat Inventory Control: Methodologies and Revenue Impacts," Ph.D. thesis, Flight Transportation Laboratory, Massachusetts Institute of Technology, Cambridge, Mass.

[45] Wollmer, R.D. 1992. "An Airline Seat Management Model for a Single Leg Route when Lower Fare Classes Book First," Oper. Res. 40, 26-37.

[46] Wu, C.F.J. 1983. "On the Convergence Properties of the EM Algorithm," Annals of Stat., 11, 95-103.

[47] Young, Y. and R. Van Slyke 1994. "Stochastic Knapsack Models of Yield Management," Technical Report, 94-76, Brooklyn Polytechnic University, 1994.

[48] Zhao, W. 1999. "Dynamic and Static Yield Management Models", Ph.D. thesis, The Wharton School, Operations and Information Management Department, University of Pennsylvania, Philadelphia. 